\title{
Effectiveness of noninvasive brain stimulation in the treatment of anxiety disorders: a meta-analysis of sham or behaviour-controlled studies
}

\author{
Alessandra Vergallito, PhD; Alessia Gallucci, MA; Alberto Pisoni, PhD; \\ Mariacristina Punzi, MA; Gabriele Caselli, PhD; Giovanni M. Ruggiero, MD; \\ Sandra Sassaroli, MD; Leonor J. Romero Lauro, PhD
}

\begin{abstract}
Background: The possibility of using noninvasive brain stimulation to treat mental disorders has received considerable attention recently. Repetitive transcranial magnetic stimulation (rTMS) and transcranial direct current stimulation (tDCS) are considered to be effective treatments for depressive symptoms. However, no treatment recommendation is currently available for anxiety disorders, suggesting that evidence is still limited. We conducted a systematic review of the literature and a quantitative analysis of the effectiveness of $r$ TMS and tDCS in the treatment of anxiety disorders. Methods: Following PRISMA guidelines, we screened 3 electronic databases up to the end of February 2020 for English-language, peer-reviewed articles that included the following: a clinical sample of patients with an anxiety disorder, the use of a noninvasive brain stimulation technique, the inclusion of a control condition, and pre/post scores on a validated questionnaire that measured symptoms of anxiety. Results: Eleven papers met the inclusion criteria, comprising 154 participants assigned to a stimulation condition and 164 to a sham or control group. We calculated Hedge's $g$ for scores on disorder-specific and general anxiety questionnaires before and after treatment to determine effect size, and we conducted 2 independent random-effects metaanalyses. Considering the well-known comorbidity between anxiety and depression, we ran a third meta-analysis analyzing outcomes for depression scores. Results showed a significant effect of noninvasive brain stimulation in reducing scores on disorder-specific and general anxiety questionnaires, as well as depressive symptoms, in the real stimulation compared to the control condition. Limitations: Few studies met the inclusion criteria; more evidence is needed to strengthen conclusions about the effectiveness of noninvasive brain stimulation in the treatment of anxiety disorders. Conclusion: Our findings showed that noninvasive brain stimulation reduced anxiety and depression scores compared to control conditions, suggesting that it can alleviate clinical symptoms in patients with anxiety disorders.
\end{abstract}

\section{Introduction}

Anxiety disorders are the most prevalent class of mental disorder in most Western societies ${ }^{1,2}$ and are one of the foremost causes of disability. ${ }^{3}$ (For epidemiologic details, see Craske and colleagues. ${ }^{4}$ ) The onset of anxiety disorders typically occurs in young adulthood. ${ }^{5}$ Then, they seem to take a chronic course, characterized by remitted and relapsed periods; the stability of the disease across time varies among studies and specific diagnoses. ${ }^{4,6}$

According to DSM-5, 7 anxiety disorders include specific phobias, social anxiety disorder, panic disorder, agoraphobia and generalized anxiety disorder (posttraumatic stress disorder and obsessive-compulsive disorders no longer fall in this grouping, and have not been considered in this meta- analysis). The DSM-5 diagnostic criteria for anxiety are similar to those of the other standard classification system, the International Classification of Diseases, tenth edition (ICD-10). ${ }^{8}$ In both systems, anxiety disorders are a spectrum of multidimensional phenotypes ${ }^{9}$ that share clinical features, such as excessive and stable anxiety; physiologic symptoms, such as tachycardia and chest tightness; and typical behavioural responses, such as avoiding perceived threats, places or situations, that impair people's psychological well-being and quality of life.

The neurobiology of anxiety disorders is still unclear. Studies have been conducted with small participant samples, and with heterogeneous imaging methods, paradigms and patient comorbidities. ${ }^{10}$ Although disease-specific differences exist, converging evidence suggests that in general, anxiety

Correspondence to: A. Pisoni, Department of Psychology, University of Milano-Bicocca, Piazza dell'Ateneo Nuovo 1, 20126 Milano, Italy; alberto.pisoni@unimib.it

Submitted Mar. 29, 2021; Revised May 23, 2021; Accepted Jul. 2, 2021

Cite as: J Psychiatry Neurosci 2021 November 9;46(6). doi: 10.1503/jpn.210050 
disorders are characterized by structural and functional alterations that primarily involve a mesocorticolimbic pathway (see Duval and colleagues ${ }^{11}$ for a review). According to this neurobiological account, the amygdala, the prefrontal cortex, the anterior cingulate cortex, the hippocampus and their functional connections might play a key role in generating and regulating fear, anxiety and threat detection. ${ }^{4,9}$ Hyperactivity in the amygdala is one of the most consistent findings. ${ }^{12,13}$ Such abnormal activity has been reported across several specific diseases and tasks, such as anxiety-provoking public speaking, ${ }^{14-16}$ fear-conditioning ${ }^{17,18}$ or presentation tasks that involve emotional images or threatening faces in social phobia or social anxiety disorder. ${ }^{19,20}$ Moreover, activation of the amygdala has been positively correlated with symptom severity ${ }^{21,22}$ and decreases after intervention with medication and psychotherapy. ${ }^{22-25}$ The response of the amygdala to threat is regulated via bidirectional connections to the anterior cingulate cortex and ventromedial prefrontal cortex in animals and humans. ${ }^{26,27}$ In line with this finding, human neuroimaging studies have highlighted hypoactivity in the prefrontal cortex in anxious patients, suggesting that amygdala hyperactivity might be the result of a decrease in top-down inhibitory control exerted by the prefrontal cortex $^{28-31}$ (but see Kraus and colleagues ${ }^{32}$ for different results). Considering the functional abnormalities seen in anxiety disorders, it has been suggested that an interhemispheric imbalance might be at their basis, involving hypoactivation of the left dorsolateral prefrontal cortex (dlPFC) and hyperactivation of the right dlPFC. ${ }^{33-35}$

First-line treatments for anxiety comprise pharmacological or psychotherapeutic interventions; cognitive-behavioural therapy is considered the most effective treatment, according to several international guidelines. ${ }^{36,37}$ However, a consistent number of patients fail to respond to traditional treatment or experience relapse and recurrence of their symptoms. ${ }^{38,39}$ In the search for alternative treatments over the last 30 years, interest in the use of noninvasive brain stimulation has grown rapidly, as a standalone therapy or combined with cognitive or behavioural interventions. ${ }^{40-42}$ The rationale for using noninvasive brain stimulation in psychiatric treatment is the possibility of rebalancing maladaptive activity and functional connectivity between brain structures. Indeed, there is a consensus that in addition to genetic, hormonal, social and cognitive factors, psychiatric disorders also involve pathologically altered neural plasticity, which can be modulated through noninvasive brain stimulation, with biochemical effects that outlast the time of stimulation. $^{43}$ (For recent reviews, see Kronberg and colleagues ${ }^{44}$ and Ziemann. ${ }^{45}$ ) Although the precise mechanisms of action are still under investigation, the effects of noninvasive brain stimulation on synaptic plasticity involve several phenomena, ultimately leading to long-term potentiation (synaptic strengthening) and long-term depression (or synaptic weakening) processes. ${ }^{46}$ (For a review and discussion, see Cirillo and colleagues. ${ }^{47}$ )

Among noninvasive brain stimulation techniques, the 2 most commonly used are transcranial magnetic stimulation (TMS) and transcranial direct current stimulation (tDCS). TMS is a technique based on delivering a strong, short magnetic pulse to the patient's head, inducing neuronal firing by suprathreshold neuronal membrane depolarization. ${ }^{48}$ When used to generate long-term effects, TMS is typically applied using repetitive (rTMS) protocols, with inhibitory $(\leq 1 \mathrm{~Hz}$ and continuous theta-burst stimulation) or excitatory $(>5 \mathrm{~Hz}$ and intermittent theta-burst stimulation [iTBS]) protocols. ${ }^{49} \mathrm{tDCS}$ is a neuromodulatory technique in which weak constant direct current (typically 1-2 mA) is delivered through the scalp using 2 electrodes, 1 with a positive (anode) polarity and 1 with a negative (cathode) polarity..$^{50}$ tDCS does not generate action potentials per se, but it does induce small changes at the membrane potential level, influencing spike frequency and, in turn, cortical excitability. ${ }^{51,52}$ The effects of tDCS are polaritydependent: anodal stimulation depolarizes the neuronal membrane and cathodal stimulation hyperpolarizes it, increasing and decreasing cortical excitability, respectively. ${ }^{53}$

Of the parameters for noninvasive brain stimulation, stimulation frequency for TMS (high or low) and polarity for tDCS (either anodal or cathodal) are usually considered the determinants of an expected effect in cortical excitability and behaviour: excitatory-enhancing or inhibitory-disrupting. Although a detailed discussion of the 2 techniques goes beyond the scope of this meta-analysis, it is crucial to point out that such an expectation can be misleading. Indeed, the outcomes of noninvasive brain stimulation - in terms of both cortical excitability and behavioural modulation - cannot be clearly determined in advance. They are the result of more complex interactions involving stimulation parameters (intensity, orientation), cerebral regions and their connections, individual anatomic features, and state dependency. ${ }^{54-57}$

Among psychiatric disorders, the main field in which noninvasive brain stimulation is applied as an alternative treatment is major depressive disorder (MDD). The clinical use of rTMS to treat MDD was approved by the US Food and Drug Administration in 2008 using high-frequency $(10 \mathrm{~Hz})$ left-side stimulation of the dlPFC and in 2018 using iTBS over the same region. ${ }^{58}$ The effectiveness of rTMS and tDCS for other psychiatric disorders has been explored in several reviews and meta-analyses targeting schizophrenia, ${ }^{59,60}$ substance abuse $^{61}$ and obsessive-compulsive disorder ${ }^{62,63}$ with promising but preliminary results.

To provide shared recommendations for good practice, periodically updated guidelines from independent expert panels have reviewed and analyzed studies investigating rTMS $^{64,65}$ and tDCS ${ }^{66,67}$ protocols for a broad spectrum of neurologic and psychiatric disorders. According to the guidelines' levels of classification, level A ("definitely effective or ineffective") indicates that the evidence was sufficient (in terms of number and quality of studies) to establish whether or not a specific protocol applied over a certain region was useful for a particular disorder. Only a few protocols have reached level A. For TMS, the protocols with level A effectiveness are as follows: high-frequency rTMS applied to the left dlPFC to treat depression, high-frequency TMS to the primary motor cortex contralateral to the painful side for neuropathic pain, and low-frequency rTMS applied over the contralesional primary motor cortex for hand motor recovery in the postacute stage of stroke. ${ }^{65}$ For tDCS, level A effectiveness has 
been assigned only to anodal stimulation over the left dlPFC in depression; ${ }^{66}$ anodal tDCS over the ipsilesional primary motor cortex is considered definitely not effective for enhancing robotic therapy in motor rehabilitation for subacute stroke. To date, no recommendation has been made for the use of rTMS or tDCS in the treatment of anxiety disorders; the available data are not sufficient to make recommendations, either for its use or to claim an absence of effect. ${ }^{65,66}$

To fill this gap in the literature, several recent reviews have examined the available literature related to the therapeutic effects of rTMS and tDCS in the treatment of anxiety disorders, ${ }^{35,68}$ anxiety symptoms arising from other pathologies ${ }^{69}$ and specific anxiety disorders (e.g., generalized anxiety disor$\left.\operatorname{der}^{70}\right)$. These reviews testify to the general interest in this topic and have shown promising yet preliminary results. However, so far they have included single-case studies and protocols without a control condition, providing an overview of the state of the art, but without cumulatively quantifying the results. To our knowledge, 3 meta-analyses ${ }^{71-73}$ have investigated the efficacy of rTMS from a quantitative perspective. Cui and colleagues $^{72}$ investigated the efficacy of rTMS in treating generalized anxiety disorder. They included 21 studies (2 in English and 19 in Chinese), all with a control group receiving sham rTMS or no intervention, suggesting that rTMS was a useful option for decreasing the symptoms of generalized anxiety disorder. Trevizol and colleagues ${ }^{73}$ investigated the efficacy of rTMS in randomized clinical trials of anxiety disorders. Their review included 14 papers, but 5 of those investigated postraumatic stress disorder and 8 investigated obsessivecompulsive disorder, both of which are now considered independent diagnostic categories. ${ }^{7}$ The authors concluded that TMS was not superior to the sham condition in reducing anxiety symptoms. In line with this, Cirillo and colleagues ${ }^{71}$ conducted a systematic review and analysis in anxiety and posttraumatic stress disorder that included 17 papers: 9 considering posttraumatic stress disorder, 2 specific phobias, 2 panic disorder, and 4 generalized anxiety disorder. The authors ran 2 independent meta-analyses: 1 for posttraumatic stress disorder and 1 for generalized anxiety disorder. They considered the mean difference in pre- and post-treatment scores for sham stimulation versus TMS when the 2 conditions were available, and for the mean difference in pre/post scores for TMS when sham stimulation was not tested. The results showed substantial treatment efficacy for both disorders.

To our knowledge, no previous meta-analyses have combined TMS and tDCS to investigate the effectiveness of noninvasive brain stimulation in treating anxiety disorders. Moreover, some of the previous reports included research that did not involve a control group or involved disorders that are now considered to be separate nosological entities. In the present study, we aimed to qualitatively assess and quantitatively evaluate the effect of rTMS and tDCS protocols in anxiety disorders. We also aimed to overcome the limitations of individual studies, which have been typically conducted using small sample sizes and applying heterogeneous stimulation parameters and different numbers of sessions. ${ }^{35}$

Similar to anxiety, a neurobiological pattern of imbalance of cortical excitability between the right and left dIPFC has been reported in $\mathrm{MDD},{ }^{74-76}$ in line with the frequent comorbidity of the 2 disorders. ${ }^{77-79}$ Indeed, although anxiety and depression have been considered to be nosologically independent categories according to traditional classifications, their comorbidity is common, with reported overlap rates of $40 \%-50 \%$ (see Choi and colleagues ${ }^{80}$ and Ionescu and colleagues ${ }^{81}$ for reviews). However, despite this clinical evidence, the comorbidity between anxiety and depression is often overlooked in the literature, possibly because of a lack of a clear and noncontroversial definition (see Ionescu and colleagues ${ }^{81}$ ). We reasoned that the stimulation of brain areas involved in anxiety and mood disorders might also produce changes in depressive symptom scores; for this reason, we included studies in which patients had an additional depression diagnosis in our meta-analysis and when pre/post scores from depressive symptoms questionnaires were available, we analyzed those as well.

\section{Methods}

We used the Preferred Reporting Items for Systematic Reviews and Meta-Analyses (PRISMA) guidelines ${ }^{82,83}$ to conduct this systematic review and meta-analysis (Appendix 1, available at jpn.ca).

\section{Literature search}

We used PubMed, Web of Science and Scopus to select peerreviewed original papers published in English before the end of February 2020, exploring the application of rTMS or tDCS in patients with anxiety disorders. We combined key words for brain stimulation techniques ("rTMS," "tDCS") with relevant anxiety disorder labels ("generalized anxiety disorder," "agoraphobia," "panic disorder," "specific phobia," "social anxiety"). We excluded non-English papers, case reports, systematic and narrative reviews, meta-analyses, conference proceedings and abstracts. We also excluded reports that measured anxiety in nonclinical populations, reports without a sham or behavioural control condition, and reports without at least 1 validated clinical questionnaire. When multiple papers were based on the same data set, we included only the oldest paper reporting the results relevant to our metaanalysis - namely the effect of stimulation treatment on anxiety and depressive symptom questionnaire scores.

\section{Records screening and data extraction}

To blind the screening process, we used Rayyan (rayyan.qcri. org/), a web and mobile systematic reviews manager. ${ }^{84}$ After duplicates had been removed, 3 researchers (A.V., A.G., A.P.) independently categorized the records as "include," "exclude" or "maybe" based on titles and abstracts. Reasons for exclusion were specified by defined labels based on the inclusion and exclusion criteria. Then, the same 3 researchers analyzed the full texts of the remaining records and independently selected eligible studies. When the full versions of articles were not available, we contacted the corresponding authors. In both the titleabstract and full-text screening phases, conflicting decisions were solved by consensus. One researcher (A.P.) extracted data 
using a structured form, and the data were checked for consistency and accuracy by the other 2 authors (A.V., A.G.). Discrepancies were resolved by consensus.

We extracted anxiety and depression questionnaire scores for the treatment and control groups from the included studies. Because at least 2 measures of anxiety were available for each study, we decided to code them in 2 separate metaanalyses. The first meta-analysis targeted questionnaire scores that investigated symptoms specific to the disorder for which participants were included in the study (e.g., for panic disorder, "Please indicate how many panic and limited symptoms attacks did you have during the week?"). The second meta-analysis considered a more general core typically related to all anxiety symptoms (e.g., "Please indicate how much you were bothered by feeling unable to relax in the last month."). When measures of depressive symptoms were available, we also collected these.

\section{Study quality assessment}

Two researchers (A.V., A.G.) independently assessed the quality of the studies based on the criteria in the Cochrane Collaboration's risk-of-bias tool: ${ }^{85}$ random sequence generation, allocation concealment, blinding strategy, incomplete outcome data and selective outcome reporting. To determine selection bias, we rated random sequence generation as low-risk only when randomization procedures were reported (e.g., random number table, computer-generated randomization, randomization envelopes). We rated allocation concealment as low-risk only for studies that recruited a group of patients who received sham stimulation. To determine reporting bias, we checked the registered protocol of the included records when available. Conflicts were solved by consensus of the 2 researchers and by consulting a third researcher when needed (A.P.).

\section{Quantitative analysis}

For each included study, we extracted relevant information, including means and standard deviations of scores on clinical scales, the noninvasive brain stimulation protocol (technique, number of sessions, stimulation location), and patient characteristics. As the primary outcome measure, we extracted the pre/post-treatment mean difference in anxiety-disorderspecific scales (10 studies included) for the treatment and control groups to measure the effect of the noninvasive brain stimulation protocol on anxiety symptoms. When information in the text, tables or supplementary material was insufficient, we contacted the authors to obtain missing data. ${ }^{86-88} \mathrm{We}$ calculated the standard deviation (SD) of the change score (pre- to post-noninvasive brain stimulation treatment), as suggested by the Cochrane Handbook for Systematic Reviews of Interventions: ${ }^{89}$

$$
S D_{\text {change }}=\sqrt{S D_{\text {pre }}^{2}+S D_{\text {post }^{2}}-\left(2 \times \text { corr } \times S D_{\text {pre }} \times S D_{\text {post }}\right)}
$$

where corr was the correlation between pre- and postmeasurement variances, set at 0.5 as suggested by Follman and colleagues..$^{90}$
We computed sampling variance, standardized mean difference (SMD) and summary analyses for each included study, using the "escalc" function of the "metafor" package in R (version 3.4.3) ${ }^{91,92}$ We corrected SMD for positive bias for a small group within the function, calculating Hedge's $g^{9}{ }^{93}$ which we used as a measure of effect size. We calculated the global effect of noninvasive brain stimulation in reducing anxiety symptoms using a random-effects model with the "rma" function of the "metafor" package in R. Randomeffects meta-analyses can account for heterogeneity due to sampling errors and large variations in effect size. ${ }^{94}$ Studies vary in their characteristics (e.g., patient characteristics, stimulation interventions, associated therapies), and this influences the effect size. Therefore, the included studies represent only a portion of the possible population of studies to be performed. This motivated our decision to include random effects in our analyses. We also assessed heterogeneity through variation because of sampling errors ( $Q$ statistic) and the percentage of variation between studies because of heterogeneity rather than chance $\left(I^{2}\right.$ statistics $) .{ }^{95}$ We identified potential outliers with an analysis of influence, ${ }^{96-98}$ implemented using the "inf" function of the "metafor" package in R. We controlled for publication bias using funnel plots, Egger's regression test ${ }^{99}$ and the rank correlation test, ${ }^{100}$ and eventually corrected any bias using the "trim and fill" method, ${ }^{101}$ which creates dummy potential missing studies to create a more symmetric funnel plot.

Finally, we ran an exploratory moderation analysis. In noninvasive brain stimulation, some features are crucial for the final outcome, such as the number of sessions, the type of stimulation, the interaction between brain areas and the type of stimulation. However, given the limited number of studies in the meta-analysis, we could not include all of the potentially interesting moderators. Therefore, we included only the informative ones - namely those that were sufficiently represented in the selected papers. We adopted the same procedures for the secondary outcome measures: general anxiety scale (Hamilton Anxiety Rating Scale [HAM-A] or Beck Anxiety Inventory [BAI]; 9 studies included) and depressive interview / self-report questionnaire (Hamilton Depression Rating Scale [HAM-D] or Beck Depression Inventory; 7 studies).

\section{Results}

\section{Study selection}

We identified a total of 876 publications. We removed 239 duplicates and carefully reviewed the titles and abstracts of the remaining 637 records. Of these, we excluded 611 records because they did not meet our inclusion criteria. We then examined the full texts of the remaining 26 papers and excluded 15 records at this stage. We excluded 6 studies because they did include a control condition (sham stimulation or a control group). ${ }^{102-107}$ We excluded 5 studies because they involved samples already analyzed in previous articles. (When multiple reports were published on the same study sample, we included reports according to publication date; the oldest paper was always included in our analyses. We also checked that subsequent reports did not increase the 
sample size, only adding secondary analysis to the original sample). ${ }^{108-112}$ We excluded 1 paper because it did not include patients, ${ }^{29} 1$ because it did not include patients with a diagnosis of an anxiety disorder, ${ }^{112} 1$ because it did not test anxiety as an outcome measure, ${ }^{113}$ and 1 because the full text was not available. ${ }^{114}$ Figure 1 summarizes the selection procedure.

Overall, 11 studies met our inclusion criteria and were included in the meta-analysis. Study characteristics are shown in Table 1.

\section{Study quality assessment}

Results of the quality assessment are reported in Table 2. We calculated the percentage of high-risk judgments to obtain a quality score for each study. The average quality of the included studies was high to intermediate (range $0 \%$ to $42.86 \%$ ); random sequence generation was the primary source of methodological bias, followed by blinding mode. Most of the studies did not describe randomization procedures, and 2 studies employed a single-blind design..$^{88,120}$ Three studies $^{87,88,115}$ reported confusing information about the number of patients excluded from the final sample analyzed.

We evaluated reporting bias based on the details reported in the full text, except for 3 studies ${ }^{86,116,121}$ whose registered protocol was available to check the completeness and consistency of the findings. Of these, Nasiri and colleagues ${ }^{121}$ did not report analyses and results for some of the preregistered outcome variables, because the report was part of a larger project. Concerning allocation concealment, only Nasiri and colleagues $^{121}$ received a high-risk judgment because they used cognitive treatment and not sham stimulation as a control condition.

\section{Participant characteristics and inclusion/exclusion criteria}

Eleven studies were included in the meta-analysis, involving 154 participants assigned to stimulation groups and 164 to control groups. Participants' ages ranged from 18 to 65 years; when reported (10 out of 11 studies), participants' mean age $( \pm \mathrm{SD})$ was $36.4 \pm 6.6$ years for the stimulation groups and $36.8 \pm 7.2$ years for the control groups. In most studies, the number of females was greater than the number of males, and secondary school was the most common education level. Specific participant characteristics from the included studies are reported in Table 3 .

The studies differed in terms of number of stimulation sessions (1-25 sessions), intervention techniques (rTMS, tDCS or iTBS), the presence or absence of concomitant treatments (pharmacological or psychological interventions) and patient diagnoses.

Inclusion and exclusion criteria differed across studies. Participants were typically included if they were in a certain age range, had a specific diagnosis (according to standardized diagnostic manuals such as the Diagnostic and Statistical
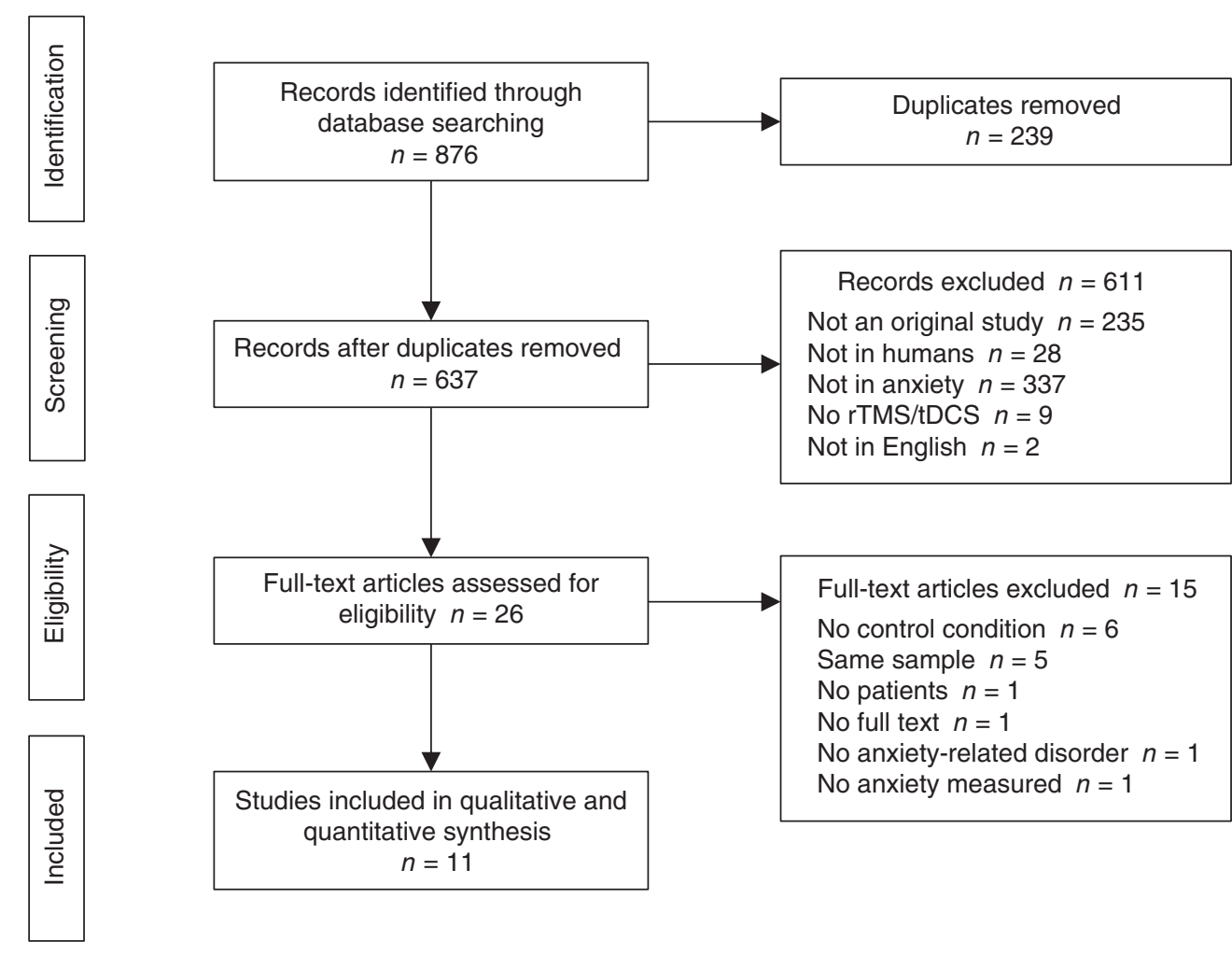

Figure 1: Flow chart of study selection. rTMS = repetitive transcranial magnetic stimulation; tDCS = transcranial direct current stimulation. 
Table 1: Summary of study characteristics used for quantitative analysis

\begin{tabular}{|c|c|c|c|c|c|c|c|c|c|c|c|}
\hline \multirow[b]{3}{*}{ Study } & \multicolumn{8}{|c|}{ Sample } & \multicolumn{3}{|c|}{ Outcome measures } \\
\hline & \multicolumn{2}{|c|}{ NIBS } & \multicolumn{2}{|c|}{ Control } & \multirow[b]{2}{*}{$\begin{array}{c}\text { No. } \\
\text { sessions }\end{array}$} & \multirow[b]{2}{*}{$\begin{array}{l}\text { Target } \\
\text { region }\end{array}$} & \multirow[b]{2}{*}{$\begin{array}{l}\text { Protocol } \\
\text { type }\end{array}$} & \multirow[b]{2}{*}{ Blinding } & \multirow[b]{2}{*}{$\begin{array}{l}\text { Specific } \\
\text { anxiety }\end{array}$} & \multirow[b]{2}{*}{$\begin{array}{l}\text { General } \\
\text { anxiety }\end{array}$} & \multirow[b]{2}{*}{ Depression } \\
\hline & Type & $\begin{array}{l}\text { No. } \\
\text { patients }\end{array}$ & Type & $\begin{array}{c}\text { No. } \\
\text { patients }\end{array}$ & & & & & & & \\
\hline $\begin{array}{l}\text { De Lima et al. }{ }^{86} \\
(2019)\end{array}$ & tDCS & 15 & Sham & 15 & 5 & $\begin{array}{l}\text { Left } \\
\text { dIPFC }\end{array}$ & Excitatory & Double-blind & Lipp & HAM-A & $\mathrm{BDI}$ \\
\hline $\begin{array}{l}\text { Deppermann et } \\
\text { al. }^{115}(2014)\end{array}$ & iTBS & 20 & Sham & 21 & 15 & $\begin{array}{l}\text { Left } \\
\text { dIPFC }\end{array}$ & Excitatory & Double-blind & PAS & HAM-A & NR \\
\hline $\begin{array}{l}\text { Diefenbach et } \\
\text { al. }{ }^{116}(2016)\end{array}$ & rTMS & 9 & Sham & 10 & 10 & $\begin{array}{l}\text { Right } \\
\text { dIPFC }\end{array}$ & Inhibitory & Double-blind & PSWQ & HAM-A & HAM-D \\
\hline $\begin{array}{l}\text { Dilkov et al. }{ }^{87} \\
(2017)\end{array}$ & rTMS & 15 & Sham & 22 & 25 & $\begin{array}{l}\text { Right } \\
\text { dIPFC }\end{array}$ & Excitatory & Double-blind & NR & HAM-A & HAM-D \\
\hline $\begin{array}{l}\text { Herrmann et } \\
\text { al. }^{117}(2017)\end{array}$ & rTMS & 20 & Sham & 19 & 2 & vmPFC & Excitatory & Double-blind & $A Q$ anxiety & NR & NR \\
\hline $\begin{array}{l}\text { Huang et al. }{ }^{118} \\
(2018)\end{array}$ & rTMS & 18 & Sham & 18 & 10 & $\begin{array}{l}\text { Right } \\
\text { PPC }\end{array}$ & Inhibitory & Double-blind & PSQI & HAM-A & HAM-D \\
\hline $\begin{array}{l}\text { Mantovani et } \\
\text { al. }{ }^{119}(2013)\end{array}$ & rTMS & 11 & Sham & 10 & 20 & $\begin{array}{l}\text { Right } \\
\text { dIPFC }\end{array}$ & Inhibitory & Double-blind & PDSS & HAM-A & HAM-D \\
\hline $\begin{array}{l}\text { Movahed et al. }{ }^{120} \\
(2018)\end{array}$ & tDCS & 6 & Sham & 6 & 10 & $\begin{array}{l}\text { Right } \\
\text { dIPFC }\end{array}$ & Inhibitory & Single-blind & PSWQ & HAM-A & HAM-D \\
\hline $\begin{array}{l}\text { Nasiri et al. }{ }^{121} \\
(2020)\end{array}$ & tDCs & 13 & UP & 15 & 10 & $\begin{array}{l}\text { Right } \\
\text { dIPFC }\end{array}$ & Inhibitory & Double-blind & GAD-Q-IV & BAI & $\mathrm{BDI}$ \\
\hline $\begin{array}{l}\text { Notzon et al. }{ }^{88} \\
(2015)\end{array}$ & iTBS & 20 & Sham & 20 & 1 & $\begin{array}{l}\text { Left } \\
\text { dIPFC }\end{array}$ & Excitatory & Single-blind & $\mathrm{SPQ}$ & NR & NR \\
\hline $\begin{array}{l}\text { Prasko et al. }{ }^{122} \\
(2007)\end{array}$ & rTMS & 7 & Sham & 8 & 10 & $\begin{array}{l}\text { Right } \\
\text { dIPFC }\end{array}$ & Inhibitory & Double-blind & PDSS & HAM-A & NR \\
\hline $\begin{array}{l}\text { AQ anxiety = Acroph } \\
\text { Generalized Anxiety } \\
\text { Lipp = Lipp Inventor } \\
\text { Scale; PPC = poster } \\
\text { Spider Phobia Ques }\end{array}$ & $\begin{array}{l}\text { ia Quest } \\
\text { sorder Q } \\
\text { f Stress } \\
\text { parietal } \\
\text { nnaire; th }\end{array}$ & $\begin{array}{l}\text { nnaire anxie } \\
\text { estionnaire-- } \\
\text { Imptoms for } \\
\text { ortex; PSQI } \\
\text { S = transcr }\end{array}$ & $\begin{array}{l}\text { ubscale; } \\
\text { IAM-A = } \\
\text { Its; NIBS } \\
\text { tsburgh } \\
\text { I direct } \mathrm{C}\end{array}$ & $\begin{array}{l}\text { Al = Beck Ar } \\
\text { Imilton Anxi } \\
\text { noninvasiv } \\
\text { ep Quality } \\
\text { ent stimulat }\end{array}$ & $\begin{array}{l}\text { iety Inventor } \\
\text { y Rating Sca } \\
\text { brain stimula } \\
\text { dex; PSWQ } \\
\text { n; UP = unifi }\end{array}$ & $\begin{array}{l}\mathrm{BDI}=\mathrm{Beck} \\
\text {; HAM-D = } \\
\text { on; NR = no } \\
\text { Penn State } \\
\text { d protocol; v }\end{array}$ & $\begin{array}{l}\text { Depression In } \\
\text { amilton Depre } \\
\text { reported; PAS } \\
\text { Vorry Question } \\
\text { IPFC = ventro }\end{array}$ & $\begin{array}{l}\text { entory; dIPFC = I€ } \\
\text { sion Rating Scale } \\
\text { = Panic and Agor } \\
\text { laire; rTMS = rep } \\
\text { edial prefrontal c }\end{array}$ & $\begin{array}{l}\text { dorsolateral pre } \\
\text { iTBS = intermitt } \\
\text { hobia Scale; P } \\
\text { itive transcrania }\end{array}$ & $\begin{array}{l}\text { ntal cortex; } \\
\text { theta burst } \\
=\text { Panic D } \\
\text { agnetic stim }\end{array}$ & $\begin{array}{l}\text { D-Q-IV = } \\
\text { nulation; } \\
\text { der Severity } \\
\text { tion; SPQ = }\end{array}$ \\
\hline
\end{tabular}

Table 2: Risk of bias among included studies

\begin{tabular}{|c|c|c|c|c|c|c|c|c|}
\hline \multirow[b]{3}{*}{ Study } & \multicolumn{7}{|c|}{ Cochrane Items } & \multirow[b]{3}{*}{$\begin{array}{c}\text { No. of high, } \\
\%^{*}\end{array}$} \\
\hline & \multicolumn{2}{|c|}{ Selection bias } & \multirow{2}{*}{$\begin{array}{c}\begin{array}{c}\text { Performance } \\
\text { bias }\end{array} \\
\begin{array}{c}\text { Blinding of } \\
\text { participants } \\
\text { and personnel }\end{array}\end{array}$} & \multirow{2}{*}{$\begin{array}{c}\begin{array}{c}\text { Detection } \\
\text { bias }\end{array} \\
\begin{array}{c}\text { Blinding of } \\
\text { outcome } \\
\text { assessment }\end{array}\end{array}$} & \multirow{2}{*}{$\begin{array}{c}\text { Attrition bias } \\
\text { Incomplete } \\
\text { outcome } \\
\text { data }\end{array}$} & \multirow{2}{*}{$\begin{array}{c}\text { Reporting } \\
\text { bias }\end{array}$} & \multirow[b]{2}{*}{ Other } & \\
\hline & $\begin{array}{c}\text { Random } \\
\text { sequence } \\
\text { generation }\end{array}$ & $\begin{array}{c}\text { Allocation } \\
\text { concealment }\end{array}$ & & & & & & \\
\hline De Lima et al. ${ }^{86}$ (2019) & Low & Low & Low & Low & Low & Low & Low & 0 \\
\hline Deppermann et al. ${ }^{115}$ (2014) & High & Low & Low & Low & Unsure & Low & Low & 14.29 \\
\hline Diefenbach et al. ${ }^{116}(2016)$ & High & Low & Low & Low & Low & Low & Low & 14.29 \\
\hline Dilkov et al. ${ }^{87}$ (2017) & Low & Low & Low & Low & High & Low & Low & 14.29 \\
\hline Herrmann et al. ${ }^{117}$ (2017) & High & Low & Low & Low & Low & Low & Low & 14.29 \\
\hline Huang et al. ${ }^{118}$ (2018) & High & Low & Low & Low & Low & Low & Low & 14.29 \\
\hline Mantovani et al. ${ }^{119}(2013)$ & High & Low & Low & Low & Low & Low & Low & 14.29 \\
\hline Movahed et al. ${ }^{120}$ (2018) & High & Low & High & High & Low & Low & Low & 42.86 \\
\hline Nasiri et al. ${ }^{121}$ (2020) & High & High & Low & Low & Low & High & Low & 42.86 \\
\hline Notzon et al. ${ }^{88}(2015)$ & High & Low & High & High & Unsure & Low & Low & 42.86 \\
\hline Prasko et al. ${ }^{122}$ (2007) & High & Low & Low & Low & Low & Low & Low & 14.29 \\
\hline
\end{tabular}

*We calculated a percentage for each study, as the quotient of the number of "High" ratings and the total number of relevant items. The lower the percentage, the lower the overall risk of bias. 
Manual of Mental Disorders [DSM] and the International Classification of Diseases [ICD]) and had a certain questionnaire score. Depression comorbidity was diagnosed by the authors when patients also fulfilled the criteria for depression (according to DSM standards). Exclusion criteria typically concerned previous psychiatric history (except for the disorder being investigated) and suicidality (Table 4).

\section{Patient diagnoses}

Studies were included if participants received a primary diagnosis of an anxiety disorder, which could be comorbid with depression. Studies in which anxiety was secondary to other conditions (e.g., an organic/neurologic condition, substance use, etc.) were excluded. Participants had a diagnosis of generalized anxiety disorder in 6 of the 11 included studies ${ }^{86,87,116,118,120,121}$ - combined with insomnia in 1 of the $6^{118}$ and combined with MDD in 1 of the $6 .{ }^{121}$ In 3 studies, participants had panic disorder with or without agoraphobia ${ }^{115,119,122}-$ combined with MDD in 1 of the $3 .{ }^{119}$ The last 2 papers included participants with a specific phobia: spider phobia ${ }^{88}$ and acrophobia. ${ }^{117}$

\section{Associated therapies}

Four of 11 studies ${ }^{88,115,117,121}$ provided psychological interventions as part of treatment. In the study by Deppermann and colleagues, ${ }^{115}$ participants took part in 3 group sessions of psychoeducation about panic disorder, which occurred separately from the stimulation sessions. Nasiri and colleagues ${ }^{121}$ added noninvasive brain stimulation to the last 2 weeks of 12 weekly sessions of a unified protocol ${ }^{123}$ (the unified protocol is a transdiagnostic treatment for emotional disorders that is aimed at targeting the common features of anxiety and mood disorders using a single psychological treatment), but they did not indicate whether the stimulation was time-locked (e.g., during or immediately before) to the psychological intervention. Notzon and colleagues ${ }^{88}$ and Herrmann and colleagues ${ }^{117}$ applied noninvasive brain stimulation before exposure to virtual reality; the interventions occurred in single and double sessions, respectively. In 2 of 11 studies, ${ }^{87,119}$ individual or supportive psychotherapy was allowed during noninvasive brain stimulation sessions; in 4 of 11 studies, ${ }^{86,116,118,120}$ psychological interventions were not permitted during noninvasive brain stimulation treatment. One study ${ }^{122}$ did not report on this factor.

Table 3: Summary of participant characteristics from the included studies

\begin{tabular}{|c|c|c|c|c|c|c|c|c|}
\hline \multirow[b]{2}{*}{ Author } & \multicolumn{3}{|c|}{ Stimulation } & \multicolumn{3}{|c|}{ Control } & \multirow[b]{2}{*}{ Diagnosis } & \multirow[b]{2}{*}{ Recruitment } \\
\hline & Age, yr & $M / F$ & Education & Age, yr & $\mathrm{M} / \mathrm{F}$ & Education & & \\
\hline $\begin{array}{l}\text { De Lima et al. }{ }^{86} \\
(2019)\end{array}$ & $32.07 \pm 6.5$ & $5 / 10$ & $\begin{array}{l}2 \text { elementary, } \\
9 \text { secondary, } \\
4 \text { university }\end{array}$ & $29 \pm 5.05$ & $6 / 9$ & $\begin{array}{l}2 \text { elementary, } \\
7 \text { secondary, } \\
6 \text { university }\end{array}$ & GAD & Two outpatient clinics \\
\hline $\begin{array}{l}\text { Deppermann et } \\
\text { al. }^{115}(2014)\end{array}$ & $\begin{array}{c}37.6 \\
\text { (range 19-63) }\end{array}$ & $9 / 13^{*}$ & $12.1 \pm 1.7 \mathrm{yr}$ & $\begin{array}{c}36.3 \\
\text { (range 22-56) }\end{array}$ & $8 / 14^{*}$ & $12.4 \pm 2.0 \mathrm{yr}$ & $\begin{array}{c}\text { PD } \pm \\
\text { agoraphobia }\end{array}$ & $\begin{array}{l}\text { Outpatient } \\
\text { clinics, advertisements, } \\
\text { internet, information } \\
\text { sent to local physicians }\end{array}$ \\
\hline $\begin{array}{l}\text { Diefenbach et al. }{ }^{116} \\
(2016)\end{array}$ & $44.00 \pm 11.95$ & $1 / 8$ & $\begin{array}{l}12 \text { yr (high } \\
\text { school } \\
\text { diploma) }\end{array}$ & $44.58 \pm 14.75$ & $3 / 7$ & $\begin{array}{c}12 \text { yr (high } \\
\text { school diploma) }\end{array}$ & GAD & $\begin{array}{l}\text { Outpatient clinic, } \\
\text { advertisements, } \\
\text { internet, } \\
\text { community flyers, } \\
\text { physician referral, } \\
\text { media coverage }\end{array}$ \\
\hline Dilkov et al. ${ }^{87}$ (2017) & $34 \pm 7$ & $9 / 6$ & NR & $38 \pm 10$ & $11 / 11$ & NR & GAD & $\begin{array}{l}2 \text { mood disorder centres: } \\
\text { Canada and Bulgaria }\end{array}$ \\
\hline $\begin{array}{l}\text { Herrmann et al. }{ }^{117} \\
(2017)\end{array}$ & $43.2 \pm 12.6$ & $7 / 13$ & NR & $46.6 \pm 13.7$ & $6 / 13$ & NR & $\mathrm{SP}$ & $\begin{array}{l}\text { Advertisements in local } \\
\text { newspapers }\end{array}$ \\
\hline $\begin{array}{l}\text { Huang et al. }{ }^{118} \\
(2018)\end{array}$ & $44.94 \pm 11.64$ & $9 / 9$ & NR & $45.22 \pm 10.85$ & $9 / 9$ & NR & $\begin{array}{c}\text { GAD + } \\
\text { insomnia }\end{array}$ & $\begin{array}{l}\text { Neurology outpatient } \\
\text { clinic }\end{array}$ \\
\hline $\begin{array}{l}\text { Mantovani et al. }{ }^{119} \\
(2013)\end{array}$ & $40.2 \pm 10$ & 4/8† & NR & $39.87 \pm 13.3$ & $8 / 5 \dagger$ & NR & $\mathrm{PD}+\mathrm{MDD}$ & NR \\
\hline $\begin{array}{l}\text { Movahed et al. }{ }^{120} \\
(2018)\end{array}$ & NR & NR & NR & NR & NR & NR & GAD & NR \\
\hline Nasiri et al. ${ }^{121}(2020)$ & $20.23 \pm 2.89$ & $3 / 10$ & NR & $21.53 \pm 3.56$ & $4 / 11$ & NR & $G A D+M D D$ & $\begin{array}{l}\text { University } \\
\text { announcements }\end{array}$ \\
\hline Notzon et al. ${ }^{88}$ (2015) & $25.85 \pm 7.65$ & $20 \ddagger$ & $11.30 \pm 3.91 \mathrm{yr}$ & $27.02 \pm 9.23$ & $20 \ddagger$ & $11.34 \pm 3.51 \mathrm{yr}$ & SP & Local advertisements \\
\hline $\begin{array}{l}\text { Prasko et al. }{ }^{122} \\
(2007)\end{array}$ & $33.7 \pm 9.2$ & $1 / 6$ & $\begin{array}{l}5 \text { elementary, } \\
1 \text { secondary, } \\
1 \text { university }\end{array}$ & $33.8 \pm 12.2$ & $3 / 5$ & $\begin{array}{l}1 \text { elementary, } \\
6 \text { secondary, } \\
1 \text { university }\end{array}$ & PD & NR \\
\hline
\end{tabular}

$\mathrm{F}=$ female; $\mathrm{GAD}=$ generalized anxiety disorder; $\mathrm{M}=$ male; $\mathrm{MDD}=$ major depressive disorder; $\mathrm{NR}=$ not reported; $\mathrm{PD}=$ panic disorder; $\mathrm{SP}=\mathrm{specific}$ phobia

Values are mean \pm standard deviation or $n$, unless otherwise specified.

${ }^{*}$ The number of males and females was based on the original number of participants included in the study reported in Deppermann et al. ${ }^{110}$ (2017). Three participants did not complete the study ( 2 from the stimulation group and 1 from the sham group), but their sex was not reported by the authors.

†The number of males and females was based on the original number of participants included in the study. Four participants did not complete the study ( 1 from the stimulation group and

3 from the sham group).

łParticipant sex in the stimulation and sham groups were not specified; we have reported the total number of patients from the authors' data set. 
Four of 11 studies $88,117,120,121$ did not allow medication use during noninvasive brain stimulation treatment; the other $7^{86,87,115,116,118,121,122}$ reported that stable medication treatment was accepted. Medication stability was defined differently across the studies, ranging from 4 weeks before treatment onset $^{120}$ to 3 months before (Table 5). ${ }^{16,118}$

\section{Stimulation protocols}

Of the 11 included studies, 6 used an rTMS protocol, $, 87116-119,122$ 3 a tDCS protocol ${ }^{86,120,121}$ and 2 an iTBS protocol. ${ }^{88,115}$ In the rTMS studies, stimulation was applied at $1 \mathrm{~Hz}$ in 4 of 6 studies, ${ }^{116,118,119,122}$ at $20 \mathrm{~Hz}$ in 1 study $^{87}$ and at $10 \mathrm{~Hz}$ in 1 study. ${ }^{117}$ The target region for rTMS was the right dIPFC in 4 of the

Table 4: Inclusion and exclusion criteria for the included studies

\begin{tabular}{|c|c|c|}
\hline Study & Inclusion criteria & Exclusion criteria \\
\hline De Lima et al. ${ }^{86}(2019)$ & $\begin{array}{l}\text { GAD diagnosis (DSM-5) } \\
\text { Age } 20-30 \mathrm{yr}\end{array}$ & $\begin{array}{l}\text { Psychotherapy or hospitalization indication from the } \\
\text { psychiatrist at the beginning of the study }\end{array}$ \\
\hline Deppermann et al. ${ }^{115}$ (2014) & $\begin{array}{l}\text { Age } 18-65 \text { yr } \\
\text { PD with or without agoraphobia (DSM-IV-TR) }\end{array}$ & Severe somatic disorders \\
\hline Diefenbach et al. ${ }^{116}(2016)$ & $\begin{array}{l}\text { Age }>18 \text { yr } \\
\text { GAD as principal or coprincipal disorder } \\
\text { HAM-A and HAM-D cut-off }\end{array}$ & $\begin{array}{l}\text { Unstable medical/psychiatric condition (e.g., thyroid disease, } \\
\text { suicidality) } \\
\text { Current PTSD } \\
\text { Substance use disorder } \\
\text { Lifetime bipolar, psychotic, developmental or obsessive- } \\
\text { compulsive disorder } \\
\text { Concurrent psychotherapy }\end{array}$ \\
\hline Dilkov et al. ${ }^{87}$ (2017) & $\begin{array}{l}\text { Age } 18-65 \text { yr } \\
\text { GAD primary diagnosis (DSM-IV) }\end{array}$ & $\begin{array}{l}\text { Diagnosis of psychotic disorder, bipolar disorder I, MDD or } \\
\text { substance/alcohol dependence in the } 6 \text { months before the study } \\
\text { Severe axis II disorder } \\
\text { Suicidal } \\
\text { Severe or unstable medical conditions } \\
\text { ECT treatment in the previous } 3 \mathrm{mo} \\
\text { TMS treatment in the previous } 6 \mathrm{mo}\end{array}$ \\
\hline Herrmann et al. ${ }^{117}$ (2017) & $\begin{array}{l}\text { Specific phobia (acrophobia) diagnosis (DSM-IV) } \\
\text { Subjective motivation to do something about their fear } \\
\text { (at least } 3 \text { on a scale of } 0-10 \text {; extreme motivation) }\end{array}$ & $\begin{array}{l}\text { Heights treatment in the previous } 6 \text { mo } \\
\text { Concurrent involvement in psycho- or pharmacotherapy }\end{array}$ \\
\hline
\end{tabular}

Motion sickness with 3D movies $<4$ (scale of $0-10$ )

Age 18-65 yr

GAD primary diagnosis (DSM-IV)

Insomnia for at least 3 months

Mantovani et al. ${ }^{119}(2013)$

Movahed et al. ${ }^{120}(2018)$

Nasiri et al. ${ }^{121}(2020)$

Notzon et al. ${ }^{88}(2015)$

Prasko et al. ${ }^{122}(2007)$
Age 18-65 yr

PD and MDD primary diagnosis (DSM-IV-TR)

Current episode duration of at least a month

Residual panic attack and MDD symptoms despite medication

Stable medication for $4 \mathrm{wk}$

Stable psychotherapy for 3 mo

Age 18-55 yr

GAD diagnosis (DSM-5)

5 points or higher on the 7-item GAD scale

Age 18-40 yr

GAD primary diagnosis (DSM-5)

Comorbid MDD diagnosis (DSM-5)

No medication use

Speaks Persian fluently

Ability to participate in all assessment and treatment

sessions

Age 18-65 yr

Spider phobia (DSM-IV-TR)

At least 16 on the SPQ

ICD-10 PD with or without agoraphobia

Nonresponders to SRIs (at least $6 \mathrm{wk}$ )

Age 18-45 yr
History of psychiatric diseases except GAD

Concurrent psychotherapy or counselling

Suicide risk

History of bipolar disorder, psychotic disorder or substance dependance/abuse in the previous year

Previous mental illness

Current physical illness

Current psychological or pharmacological medication

Need for immediate medical/therapeutic intervention

Received no more than 8 sessions of CBT-based intervention

within the last $5 \mathrm{yr}$

Psychiatric disorder/substance abuse

Current diagnosis of mental disorders

Opposition to collaboration at any point in research

Suicidality

History of other psychological treatment

Severe somatic disorder

History of psychiatric disorders except for specific phobia

Psychiatric or psychotropic medication

MDD

Suicidality

HAM-D score $>16$

Organic psychiatric disorder

History of psychotic disorder in history

Abuse of alcohol or other drugs

Serious somatic disease

Using nonprescribed medication

$\mathrm{CBT}=$ cognitive behavioural therapy; DSM = Diagnostic and Statistical Manual of Mental Disorders; ECT = electroconvulsive therapy; GAD = generalized anxiety disorder; HAM-A = Hamilton Anxiety Rating Scale; HAM-D = Hamilton Depression Rating Scale; MDD = major depressive disorder; NR = not reported; PD = panic disorder; PTSD = posttraumatic stress disorder; $\mathrm{SP}=$ specific phobia; $\mathrm{SPQ}=$ Spider Phobia Questionnaire; $\mathrm{SRI}=$ serotonin reuptake inhibitor; TMS = transcranial magnetic stimulation; TR = text revision. 
6 studies, the right posterior parietal cortex in 1 study ${ }^{118}$ and the ventromedial prefrontal cortex in 1 study. ${ }^{117}$ The 2 iTBS protocols were applied over the left dlPFC. ${ }^{88,115}$ In the 3 tDCS studies, stimulation was delivered with cathodal polarity over the right dlPFC in 2 of 3 studies ${ }^{120,121}$ and with anodal polarity over the left dlPFC in 1 study. ${ }^{86}$ Overall, inhibitory protocols (cathodal tDCS, $1 \mathrm{~Hz}$ rTMS) were applied over the right dIPFC in 5 of 6 studies; only 1 targeted the right posterior parietal cortex. Facilitatory protocols (iTBS, anodal tDCS and $20 \mathrm{~Hz}$ rTMS) were delivered over the left dlPFC in 3 of 5 studies, over the right dlPFC in 1 study (see Figure 2 for a graphical representation of targeted regions) and over the ventromedial prefrontal cortex in 1 study. The stimulation intensity range in TMS studies was between $80 \%$ and $110 \%$ of the individual rest motor threshold. Magnetic pulses were delivered with figure-8-shaped coils, except for the study by Herrmann and colleagues, ${ }^{117}$ in which a round coil was used. The tDCS protocols were administered at $2 \mathrm{~mA}$ in the 3 studies, with unipolar montages and intracephalic reference in 1 of 3 studies $^{86}$ and a deltoid reference in 2 of 3 studies. ${ }^{120,121}$ Stimulation duration ranged from 10 to 30 minutes. See Table 5 and Table 6 for details.

\section{Control condition}

The presence of a control condition was an inclusion criterion for our meta-analysis. For 10 of 11 studies, this consisted of a sham condition. In 1 study, ${ }^{121}$ the control group did not receive a sham stimulation; instead, they underwent unified protocol treatment. For rTMS studies, sham stimulation was induced by varying the coil inclination at $90^{\circ}$ with respect to the stimulation site in 4 of 8 studies. ${ }^{87,88,115,122}$ In the other 4 studies, ${ }^{116,118,121,124}$ experimenters used a sham coil, which had the same appearance and produced the same noise as the real coil. Among the 3 tDCS studies, 1 applied the typical

\begin{tabular}{|c|c|c|c|c|c|c|c|c|}
\hline Study & Intensity & Duration & $\begin{array}{l}\text { Coil/electrode } \\
\text { position }\end{array}$ & $\begin{array}{l}\text { tDCS } \\
\text { reference }\end{array}$ & $\begin{array}{l}\text { Sham } \\
\text { procedure }\end{array}$ & $\begin{array}{l}\text { Psychological } \\
\text { intervention }\end{array}$ & $\begin{array}{l}\text { Treatment } \\
\text { strategy }\end{array}$ & Medication \\
\hline $\begin{array}{l}\text { De Lima et al. }{ }^{86} \\
(2019)\end{array}$ & $\begin{array}{c}2 \mathrm{~mA} \\
\text { Electrode } \\
\text { size } 5 \times 7\end{array}$ & $20 \mathrm{~min}$ & F3 & FP2 & $30 \mathrm{~s}$ & Not allowed & Monotherapy & Stable doses \\
\hline $\begin{array}{l}\text { Deppermann et al. }{ }^{115} \\
(2014)\end{array}$ & $\begin{array}{c}15 \mathrm{~Hz} \\
80 \% \mathrm{rMT}\end{array}$ & $\begin{array}{l}3 \mathrm{~min} ; 18 \\
\text { trains of } 2 \mathrm{~s}\end{array}$ & F3 & - & $\begin{array}{l}90^{\circ} \text { from } \\
\text { skull }\end{array}$ & $\begin{array}{l}\text { Psychoeducation, } \\
3 \text { group sessions }\end{array}$ & Monotherapy & $\begin{array}{l}\text { Stable doses } \\
\text { for } 3 \mathrm{wk}\end{array}$ \\
\hline $\begin{array}{l}\text { Diefenbach et al. }{ }^{116} \\
(2016)\end{array}$ & $\begin{array}{c}1 \mathrm{~Hz} \\
90 \% \mathrm{rMT}\end{array}$ & $\begin{array}{c}15 \text { min; } \\
900 \text { pulses } \\
\text { per session }\end{array}$ & $\begin{array}{c}\text { Individual } \\
\text { structural } \\
\text { MRI: } x, y, z= \\
42,36,32 \\
\text { (MNI) }\end{array}$ & - & Sham coil & Not allowed & Monotherapy & $\begin{array}{c}\text { Stable doses } \\
\text { for } 3 \text { mo or stable } \\
\text { benzodiazepines } \\
\text { for } 2 \mathrm{wk}\end{array}$ \\
\hline Dilkov et al. ${ }^{87}$ (2017) & $\begin{array}{c}20 \mathrm{~Hz} \\
110 \% \mathrm{rMT}\end{array}$ & $\begin{array}{l}20 \text { trains, } 9 \mathrm{~s} \\
\text { per train; } \\
51 \mathrm{~s} \\
\text { intertrain } \\
\text { interval }\end{array}$ & $\begin{array}{l}5 \mathrm{~cm} \text { rostral } \\
\text { to motor } \\
\text { cortex }\end{array}$ & - & $\begin{array}{l}90^{\circ} \text { from } \\
\text { skull, same } \\
\text { intensity }\end{array}$ & Allowed & Monotherapy & $\begin{array}{l}\text { Stable doses } \\
\text { for } 6 \text { mo or } \\
\text { no medications } \\
\text { for at least } 2 \mathrm{wk}\end{array}$ \\
\hline $\begin{array}{l}\text { Herrmann et al. }{ }^{117} \\
(2017)\end{array}$ & $\begin{array}{c}10 \mathrm{~Hz} \\
100 \% \mathrm{rMT}\end{array}$ & $\begin{array}{l}40 \text { trains of } \\
4 \text { s (1560 } \\
\text { pulses; } \\
\text { intertrain } \\
\text { interval } 26 \mathrm{~s}\end{array}$ & FPZ & - & Sham coil & $\begin{array}{l}\text { Virtual reality } \\
\text { exposure }\end{array}$ & Augmentation & Not allowed \\
\hline Huang et al. ${ }^{118}$ (2018) & $\begin{array}{c}1 \mathrm{~Hz} \\
90 \% \mathrm{rMT}\end{array}$ & $\begin{array}{c}3 \text { trains of } \\
500 \text { pulses; } \\
\text { intertrial } \\
\text { interval } \\
10 \text { min }\end{array}$ & P4 & - & Sham coil & Not allowed & Monotherapy & $\begin{array}{l}\text { Stable doses } \\
\text { for } 3 \mathrm{mo}\end{array}$ \\
\hline $\begin{array}{l}\text { Mantovani et al. }{ }^{119} \\
(2013)\end{array}$ & $\begin{array}{c}1 \mathrm{~Hz} \\
110 \% \mathrm{rMT}\end{array}$ & $30 \mathrm{~min}$ & $\begin{array}{l}5 \mathrm{~cm} \text { anterior } \\
\text { to motor } \\
\text { cortex }\end{array}$ & - & Sham coil & Allowed & Monotherapy & $\begin{array}{l}\text { Stable doses for } \\
4 \text { wk or no } \\
\text { medication for } \\
6-8 \text { wk before }\end{array}$ \\
\hline $\begin{array}{l}\text { Movahed et al. }{ }^{120} \\
(2018)\end{array}$ & $\begin{array}{c}2 \mathrm{~mA} \\
\text { Electrode } \\
\text { size NR }\end{array}$ & $20 \min$ & $\mathrm{F} 4$ & $\begin{array}{l}\text { Left } \\
\text { deltoid }\end{array}$ & NR & Not allowed & Monotherapy & Not allowed \\
\hline Nasiri et al. ${ }^{121}(2020)$ & $\begin{array}{c}2 \mathrm{~mA} \\
\text { Electrode } \\
\text { size } 5 \times 5\end{array}$ & $30 \mathrm{~min}$ & $\mathrm{~F} 4$ & $\begin{array}{l}\text { Left } \\
\text { deltoid }\end{array}$ & F3 & UP 12 sessions & Monotherapy & Not allowed \\
\hline Notzon et al. ${ }^{88}$ (2015) & $\begin{array}{c}15 \mathrm{~Hz} \\
80 \% \mathrm{rMT}\end{array}$ & $\begin{array}{l}3 \mathrm{~min} ; 18 \\
\text { trains of } 2 \mathrm{~s}\end{array}$ & F3 & - & $\begin{array}{l}90^{\circ} \text { from } \\
\text { skull }\end{array}$ & $\begin{array}{l}\text { Virtual reality } \\
\text { exposure }\end{array}$ & Augmentation & Not allowed \\
\hline $\begin{array}{l}\text { Prasko et al. }{ }^{122} \\
(2007)\end{array}$ & $\begin{array}{c}1 \mathrm{~Hz} \\
110 \% \mathrm{rMT}\end{array}$ & $30 \mathrm{~min}$ & $\begin{array}{l}5 \mathrm{~cm} \text { rostral } \\
\text { to motor } \\
\text { cortex }\end{array}$ & - & $\begin{array}{l}90^{\circ} \text { from } \\
\text { skull, same } \\
\text { intensity }\end{array}$ & NR & Monotherapy & Stable doses \\
\hline
\end{tabular}

$\mathrm{EEG}=$ electroencephalogram; F3 $=10-20 \mathrm{EEG}$ position corresponding to the left dorsolateral prefrontal cortex; F4 = 10-20 EEG position corresponding to the right dorsolateral prefrontal cortex; FP2 $=10-20$ EEG position corresponding to the supraorbital region; FPZ $=10-20 \mathrm{EEG}$ position corresponding to the ventromedial prefrontal cortex; $\mathrm{MNI}=\mathrm{Montreal}$ Neurological Institute; NR = not reported; P4 = 10-20 EEG position corresponding to the right posterior parietal cortex; rMT = resting motor threshold; UP = unified protocol. 

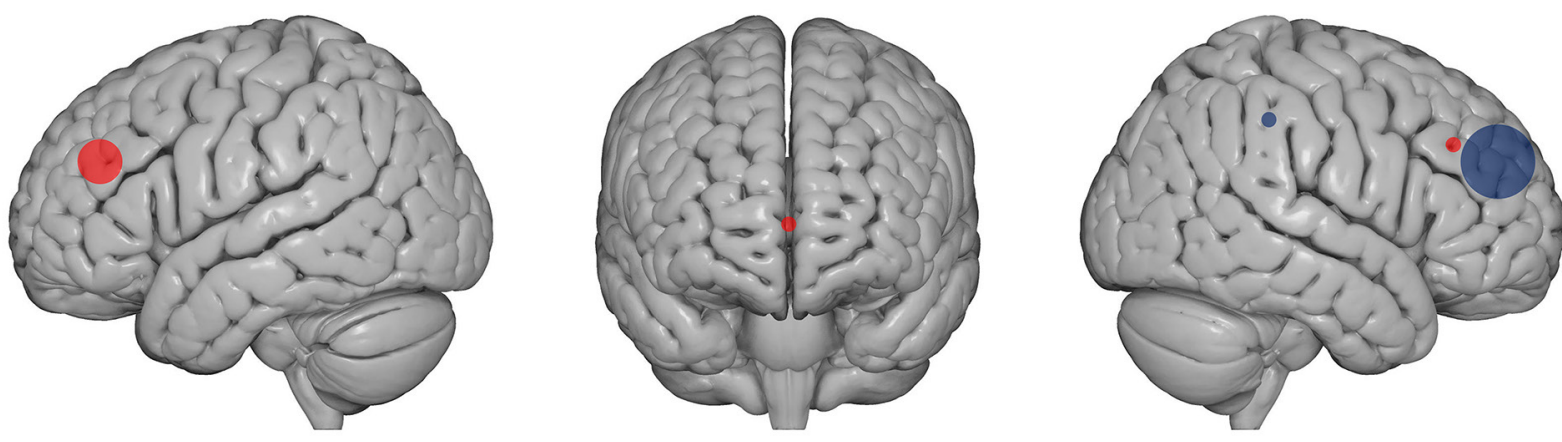

Figure 2: Type of stimulation and target regions in included studies. Red dots indicate excitatory stimulation protocols (i.e., anodal tDCS, iTBS and high-frequency rTMS); blue dots indicate inhibitory stimulation (i.e., cathodal tDCS and low-frequency rTMS). The size of the dots correspond to the number of studies that applied an excitatory or inhibitory protocol over a specific region: 5 studies applied inhibitory stimulation protocols over the right dorsolateral prefrontal cortex, 3 studies applied excitatory stimulation protocols over the left dorsolateral prefrontal cortex, 1 study applied an excitatory stimulation protocol over the right dorsolateral prefrontal cortex, 1 study applied an inhibitory stimulation protocol over the right posterior parietal cortex, and 1 study applied an excitatory stimulation protocol over the ventromedial prefrontal cortex. Brain images were obtained from www.nitrc.org. iTBS = intermittent theta burst stimulation; rTMS = repetitive transcranial magnetic stimulation; tDCS = transcranial direct current stimulation.

sham tDCS protocol: ${ }^{86}$ the stimulation was on for the first 30 seconds, inducing the same skin sensation as the real stimulation; ${ }^{125}$ Movahed and colleagues ${ }^{120}$ did not report their sham protocol parameters. Nasiri and colleagues ${ }^{121}$ did not have a sham condition; instead, they included a control group in which participants took part in cognitive treatment.

Nine of the 11 studies were double-blind, with both experimenters and participants blind to participants' assigned condition. Two of the 11 studies ${ }^{88,120}$ used a single-blind design, in which only participants were blind to their stimulation group. In 4 of 11 studies, participants' blinding was checked using specific questionnaires. ${ }^{88,115,117,119}$

\section{Outcome measures}

As noted above, we chose 3 outcome measures: an anxiety measure centred on the specific disorder investigated in each study, which was reported in 10 of 11 studies (all but Dilkov and colleagues ${ }^{87}$ ); a general anxiety measure, investigating general anxiety symptoms, reported in 9 of 11 studies (all but Notzon and colleagues ${ }^{88}$ and Herrmann and colleagues ${ }^{117}$ ); and a measure of depression, which was included in 7 of 11 studies (depression questionnaires were not included in 4 studies $\left.^{88,115,117,118}\right)$.

\section{Specific anxiety measure}

The specific anxiety outcome measure included scores from a heterogeneous pool of clinical validated questionnaires, depending on the specific disease investigated. For panic disorder, 2 of 3 studies ${ }^{119,122}$ administered the Panic Disorder Severity Scale ${ }^{126}$ and 1 study ${ }^{115}$ administered the Panic and Agoraphobia Scale. ${ }^{127}$ For the specific phobia studies, Notzon and colleagues ${ }^{88}$ used the German version of the Spider Pho- bia Questionnaire, ${ }^{128,129}$ and Herrmann and colleagues ${ }^{117}$ used the German translation of the Acrophobia Questionnaire ${ }^{130}$ anxiety subscale. For generalized anxiety disorder, the Penn State Worry Questionnaire ${ }^{131}$ was used for 2 of 6 studies, $^{116,120}$ the Generalized Anxiety Disorder Questionnaire ${ }^{132}$ was used for 1 study, ${ }^{121}$ the Lipp Inventory of Stress Symptoms for Adults ${ }^{133}$ was used for 1 study $^{86}$ and the Pittsburgh Sleep Quality Index, ${ }^{134}$ investigating insomnia symptoms, was used for 1 study. ${ }^{118}$ The final generalized anxiety disorder study ${ }^{87}$ did not include a disorder-specific questionnaire; it was not included in the specific anxiety disorders analysis.

\section{General anxiety measure}

For 8 of 9 studies we included the HAM-A, ${ }^{135}$ a 14-item clinical interview targeting somatic and psychic anxiety symptoms. For 1 of 9 studies $^{121}$ we included the BAI, ${ }^{136}$ a 21 -item selfreport questionnaire focusing on the somatic symptoms of anxiety occurring over the past week. Notzon and colleagues ${ }^{88}$ did not include a general anxiety measure; this study was not included in the analysis of general indexes of anxiety.

\section{Depression measure}

Five of 7 studies $^{87,116,118-120}$ used the HAM-D, ${ }^{137}$ a 21-item (only the first 17 aligned with the total score) clinical interview targeting somatic and neurovegetative aspects of depression. Two of 7 studies $^{86,121}$ used the Beck Depression Inventory, $^{138}$ a 21-item self-report questionnaire investigating the cognitive and affective dimensions of depression (for a comparison between HAM-D and the Beck Depression Inventory, see Brown and colleagues ${ }^{139}$ ). When both the clinical and the self-report measures of general anxiety or depression were reported, we considered only the clinicianadministered version. 
Table 6: Summary of stimulation protocol, statistical analyses, main results and additional groups and measures (part 1 of 2 )

\begin{tabular}{|c|c|c|c|c|c|c|}
\hline Study & Protocol & Follow-up & $\begin{array}{l}\text { Statistical } \\
\text { analysis }\end{array}$ & Reported results & Additional groups & $\begin{array}{c}\text { Additional pre/post } \\
\text { measures }\end{array}$ \\
\hline $\begin{array}{l}\text { De Lima et al. }{ }^{86} \\
(2019)\end{array}$ & 5 consecutive days & $1 \mathrm{wk}$ & $\begin{array}{l}\text { ANOVA } \\
\text { repeated- } \\
\text { measures }\end{array}$ & $\begin{array}{l}\text { Anxiety and depression } \\
\text { symptoms did not differ } \\
\text { between real and sham } \\
\text { tDCS. Physical symptoms of } \\
\text { stress were reduced at the } \\
\text { end of treatment and at } \\
\text { follow-up in the tDCS group } \\
\text { v. the sham group }\end{array}$ & None & $\begin{array}{l}\text { Anxiety: BAI } \\
\text { Global evaluation: } \\
\text { PANAS }\end{array}$ \\
\hline $\begin{array}{l}\text { Deppermann et } \\
\text { al. }{ }^{115}(2014)\end{array}$ & $\begin{array}{l}5 \text { daily sessions; } \\
3 \text { wk }\end{array}$ & NR & $\begin{array}{l}\text { ANOVA } \\
\text { repeated- } \\
\text { measures }\end{array}$ & $\begin{array}{l}\text { No differences in real } \\
\text { v. sham rTMS. Both groups } \\
\text { showed improvement in } \\
\text { anxiety symptoms post-iTBS } \\
\text { v. baseline }\end{array}$ & $\begin{array}{l}\text { Healthy controls; } \\
\text { only for fNIRS }\end{array}$ & $\begin{array}{l}\text { Physiological: CAQ } \\
\text { Brain activation: fNIRS } \\
\text { Cognitive: verbal fluency }\end{array}$ \\
\hline $\begin{array}{l}\text { Diefenbach et } \\
\text { al. }^{116}(2016)\end{array}$ & $\begin{array}{l}5 \text { daily sessions; } \\
6 \mathrm{wk}\end{array}$ & $\begin{array}{l}3 \text { mo, } 6 \text { mo } \\
\text { (only a } \\
\text { subset not } \\
\text { included in } \\
\text { statistical } \\
\text { analysis) }\end{array}$ & $\begin{array}{l}\text { ANOVA } \\
\text { repeated- } \\
\text { measures; } \\
\text { planned } \\
\text { contrasts }\end{array}$ & $\begin{array}{l}\text { Anxiety symptoms improved } \\
\text { in post- v. pre- } \\
\text { measurements in rTMS and } \\
\text { sham groups that persisted } \\
\text { at } 3 \text { mo follow-up only in the } \\
\text { rTMS group. Worry and } \\
\text { depressive symptoms } \\
\text { improved only in the rTMS } \\
\text { group at the end of } \\
\text { treatment and at } 3 \text { mo } \\
\text { follow-up. } \\
\text { Brain activation increased } \\
\text { after rTMS and tended to } \\
\text { decrease after sham }\end{array}$ & None & $\begin{array}{l}\text { Anxiety/mood: DASS- } \\
\text { DEP } \\
\text { Brain activation: fMRI } \\
\text { during gambling task }\end{array}$ \\
\hline $\begin{array}{l}\text { Dilkov et al. }{ }^{87} \\
(2017)\end{array}$ & $\begin{array}{l}6 \text { wk; } 5 \text { sessions/wk } \\
\text { for the first } 4 \text { wk; } \\
\text { during the wk } 5 \text {, } \\
\text { sessions reduced } \\
\text { to } 3 \text { times/wk; during } \\
\text { wk } 6 \text {, sessions } \\
\text { reduced to } \\
2 \text { times/wk }\end{array}$ & $\begin{array}{l}2 \text { wk and } 6 \\
\text { wk after the } \\
\text { end of } \\
\text { treatment }\end{array}$ & $\begin{array}{l}\text { ANOVA } \\
\text { repeated- } \\
\text { measures }\end{array}$ & $\begin{array}{l}\text { Anxiety and depressive } \\
\text { symptoms improved in the } \\
\text { stimulation v. sham } \\
\text { condition at the end of } \\
\text { treatment and the } \\
2 \text { follow-ups }\end{array}$ & None & Global evaluation: CGI \\
\hline $\begin{array}{l}\text { Herrmann et } \\
\text { al. }{ }^{117}(2017)\end{array}$ & 2 sessions & $3 \mathrm{mo}$ & $\begin{array}{l}\text { ANOVA } \\
\text { repeated- } \\
\text { measures; } \\
t \text { test }\end{array}$ & $\begin{array}{l}2 \text { sessions of rTMS reduced } \\
\text { anxiety and avoidance } \\
\text { ratings compared to the } \\
\text { sham group }\end{array}$ & None & $\begin{array}{l}\text { Anxiety: AQ-avoidance } \\
\text { subscale; BAT }\end{array}$ \\
\hline $\begin{array}{l}\text { Huang et al. }{ }^{118} \\
(2018)\end{array}$ & 10 consecutive days & $\begin{array}{l}2 \mathrm{wk} \\
1 \mathrm{mo}\end{array}$ & $\begin{array}{l}\text { ANOVA } \\
\text { repeated- } \\
\text { measures }\end{array}$ & $\begin{array}{l}\text { Anxiety, insomnia and } \\
\text { depressive symptoms } \\
\text { improved in the rTMS group } \\
\text { v. the sham group at the end } \\
\text { of treatment and the } \\
2 \text { follow-ups }\end{array}$ & None & NR \\
\hline $\begin{array}{l}\text { Mantovani et al. }{ }^{119} \\
(2013)\end{array}$ & $\begin{array}{l}5 \mathrm{~d} / \mathrm{wk} ; 4 \text { wk double- } \\
\text { blind }+4 \text { weeks real* }\end{array}$ & $\begin{array}{l}1,3 \text { and } \\
6 \mathrm{mo}\end{array}$ & $\begin{array}{l}\text { ANOVA } \\
\text { repeated- } \\
\text { measures; } \\
t \text { test }\end{array}$ & $\begin{array}{l}4 \text { weeks rTMS v. sham: } \\
\text { improvement in panic } \\
\text { symptoms but not } \\
\text { depression. } \\
8 \text { weeks of rTMS v. pre- } \\
\text { treatment: improvement in } \\
\text { panic and depressive } \\
\text { symptoms, global } \\
\text { assessment, and social } \\
\text { adjustment }\end{array}$ & None & $\begin{array}{l}\text { Anxiety: PDSS, } \\
\text { PDSS-SR } \\
\text { Mood: BDI; ZUNG-SAS } \\
\text { Global evaluation: CGI; } \\
\text { PGI; SASS }\end{array}$ \\
\hline $\begin{array}{l}\text { Movahed et al. }{ }^{120} \\
(2018)\end{array}$ & $4 w k$ & $2 \mathrm{mo}$ & $\begin{array}{l}\text { ANOVA } \\
\text { repeated- } \\
\text { measures }\end{array}$ & $\begin{array}{l}\text { Worry, anxiety and } \\
\text { depression scores were } \\
\text { reduced after cathodal tDCS } \\
\text { and pharmacotherapy v. } \\
\text { sham tDCS. } \\
\text { Pharmacotherapy was } \\
\text { stronger than tDCS in } \\
\text { reducing worry; tDCS was } \\
\text { stronger in reducing } \\
\text { depression. Anxiety } \\
\text { symptoms did not differ after } \\
\text { cathodal tDCS compared to } \\
\text { pharmacotherapy }\end{array}$ & Pharmacotherapy & NR \\
\hline
\end{tabular}


Table 6: Summary of stimulation protocol, statistical analyses, main results and additional groups and measures (part 2 of 2)

\begin{tabular}{|c|c|c|c|c|c|c|}
\hline Study & Protocol & Follow-up & $\begin{array}{l}\text { Statistical } \\
\text { analysis }\end{array}$ & Reported results & Additional groups & $\begin{array}{l}\text { Additional pre/post } \\
\text { measures }\end{array}$ \\
\hline $\begin{array}{l}\text { Nasiri et al. }{ }^{121} \\
(2020)\end{array}$ & $\begin{array}{l}10 \text { daily sessions; } \\
2 \mathrm{wk}\end{array}$ & $3 \mathrm{mo}$ & MANCOVA & $\begin{array}{l}\text { Worry, anxiety and anxiety } \\
\text { sensitivity improved after } \\
\text { UP + tDCS v. UP alone at } \\
\text { the end of treatment and at } \\
\text { follow-up }\end{array}$ & Waiting list & $\begin{array}{l}\text { Anxiety: ASI; IUS; } \\
\text { PSWQ }\end{array}$ \\
\hline $\begin{array}{l}\text { Notzon et al. }{ }^{88} \\
(2015)\end{array}$ & Single session & NR & $\begin{array}{l}\text { ANOVA } \\
\text { repeated- } \\
\text { measures }\end{array}$ & $\begin{array}{l}\text { iTBS increased sympathetic } \\
\text { activity during the spider } \\
\text { scene in both phobic and } \\
\text { healthy participants }\end{array}$ & $\begin{array}{l}\text { Healthy controls } \\
\text { (real and sham) }\end{array}$ & $\begin{array}{l}\text { Anxiety: FSQ; ASI } \\
\text { Global evaluation: IPQ; } \\
\text { SUDS; DS } \\
\text { Physiological: HR; SCL } \\
\text { Brain activation: fNIRS }\end{array}$ \\
\hline $\begin{array}{l}\text { Prasko et al. }{ }^{122} \\
(2007)\end{array}$ & $\begin{array}{l}5 \text { daily sessions; } \\
2 \mathrm{wk}\end{array}$ & $2 w k$ & $\begin{array}{l}\text { Nonparametric } \\
\text { repeated- } \\
\text { measures } \\
\text { ANOVA }\end{array}$ & $\begin{array}{l}\text { Anxiety symptoms and } \\
\text { psychopathology global } \\
\text { scores improved after both } \\
\text { real and sham rTMS }\end{array}$ & None & $\begin{array}{l}\text { Anxiety: BAI } \\
\text { Global evaluation: CGI }\end{array}$ \\
\hline \multicolumn{7}{|c|}{$\begin{array}{l}\text { ANOVA = analysis of variance; } A Q=\text { Acrophobia Questionnaire; } \mathrm{ASI}=\text { Anxiety Sensitivity Index; BAI = Beck Anxiety Inventory; BAT = Behavioral Avoidance Test; BDI = Beck Depression } \\
\text { Inventory; CAQ = Cardiac Anxiety Questionnaire; CGI = Clinical Global Impression Scale; DASS-DEP = Depression-Anxiety Scales Depression Subscale; } \mathrm{DS}=\text { = Disgust Scale; fMRI = } \\
\text { functional magnetic resonance imaging; fNIRS = functional near-infrared spectroscopy; FSQ = Fear of Spiders Questionnaire; HR = heart rate; IPQ = Igroup Presence Questionnaire; } \\
\text { iTBS = intermittent theta burst stimulation; IUS = Intolerance of Uncertainty Scale; MANCOVA = multivariate analysis of covariance; NR = not reported; PANAS = Positive and Negative } \\
\text { Affect Schedule; PDSS(-SR) = Panic Disorder Severity Scale (self-report); PGI = Patient Global Impression; PSWQ = Penn State Worry Questionnaire; rTMS = repetitive transcranial } \\
\text { magnetic stimulation; SASS = Self-reported Social Adaptation Scale; SCL = skin conductance level; SUDS = Subjective Units of Discomfort Scale; tDCS = transcranial direct current } \\
\text { stimulation; UP = unified protocol; ZUNG-SAS= Zung-Self Administered Scale. } \\
\text { *In our analysis, we included data for the baseline and the first } 4 \text { weeks of rTMS treatment. }\end{array}$} \\
\hline
\end{tabular}

Table 7: Summary of the results of the 3 meta-analyses

\begin{tabular}{|c|c|c|c|c|c|c|c|c|}
\hline Comparison & $\begin{array}{l}\text { No. of } \\
\text { studies }\end{array}$ & $\begin{array}{c}\text { Effect size summary } \\
\text { (95\% confidence interval) }\end{array}$ & $Z$ & $Q$ test & $l^{2}(\%)$ & Influence test & Egger's test & $\begin{array}{l}\text { Kendall's } \\
\text { rank test }\end{array}$ \\
\hline Specific anxiety & 9 & $\begin{array}{c}-0.4858 \\
(-0.8319 \text { to }-0.1398)\end{array}$ & $\begin{array}{c}-2.7517 \\
p=0.006\end{array}$ & $\begin{array}{l}17.6384 \\
p=0.040\end{array}$ & 48.98 & None & $\begin{array}{l}-1.2078 \\
p=0.23\end{array}$ & $\begin{array}{l}-0.2889 \\
p=0.29\end{array}$ \\
\hline General anxiety & 9 & $\begin{array}{c}0.8139 \\
(-1.4484 \text { to }-0.1794)\end{array}$ & $\begin{array}{l}-2.5142 \\
p=0.012\end{array}$ & $\begin{array}{l}41.0326 \\
p<0.001\end{array}$ & 80.50 & $\begin{array}{l}\text { Dilkov et al. }{ }^{87} \\
\quad(2017)\end{array}$ & $\begin{array}{l}-0.3108 \\
p=0.76\end{array}$ & $\begin{array}{l}-0.1667 \\
p=0.61\end{array}$ \\
\hline General anxiety* & 8 & $\begin{array}{c}-0.5684 \\
(-1.0626 \text { to }-0.0742)\end{array}$ & $\begin{array}{l}-2.2541 \\
p=0.024\end{array}$ & $\begin{array}{c}19.5887 \\
p=0.007\end{array}$ & 64.27 & None & $\begin{array}{l}-0.1009 \\
p=0.92\end{array}$ & $\begin{array}{l}-0.1429 \\
p=0.72\end{array}$ \\
\hline Depression & 7 & $\begin{array}{c}-0.9822 \\
(-1.6177 \text { to }-0.3468)\end{array}$ & $\begin{array}{l}-3.0297 \\
p=0.002\end{array}$ & $\begin{array}{c}23.4602 \\
p<0.001\end{array}$ & 74.42 & $\begin{array}{l}\text { Dilkov et al. }{ }^{87} \\
\quad(2017)\end{array}$ & $\begin{array}{l}-0.9869 \\
p=0.32\end{array}$ & $\begin{array}{l}-0.1429 \\
p=0.77\end{array}$ \\
\hline Depression* & 6 & $\begin{array}{c}-0.6433 \\
(-0.9786 \text { to }-0.3081)\end{array}$ & $\begin{array}{l}-3.7616 \\
p<0.001\end{array}$ & $\begin{array}{l}3.8846 \\
p=0.57\end{array}$ & - & None & $\begin{array}{l}-0.7960 \\
p=0.43\end{array}$ & $\begin{array}{l}-0.0667 \\
p>0.99\end{array}$ \\
\hline
\end{tabular}

*Indicates results after outlier removal.

\section{Meta-analysis}

\section{Anxiety-specific disorders}

Ten of 11 studies reported scores for specific anxiety disorder scales (Table 1). We ran a meta-analysis on these studies to compute the global effect of noninvasive brain stimulation on the reduction of anxiety-specific symptoms compared to a sham intervention. The random-effects model showed a significant medium effect of noninvasive brain stimulation on patients' symptom improvement compared to pre/post sham scores (overall SMD $-0.49,95 \%$ CI -0.83 to $-0.14 ; p=0.006$; see Table 7 for complete results and Figure 3 for a forest plot).

$Q$ statistics and $I^{2}$ suggested high heterogeneity between studies (Table 7); this may have been due to differences in methodological factors across studies. The inclusion of moderators - namely the duration of treatment (computed as the number of stimulation sessions), the stimulation technique applied (iTBS, rTMS, tDCS), the protocol type (excitatory v. inhibitory), the target region (left v. right dlPFC; Huang and colleagues $^{118}$ targeted a different region, so we did not include it when analyzing the moderation effect of the target region) and comorbid depression (presence v. absence) - was not significant (all $p>0.13$; see Table 8 for moderators' statistical results).

Baujat plot inspection ${ }^{140}$ (Figure 4) suggested that study $8^{119}$ greatly contributed to the heterogeneity of the meta-analysis. Nevertheless, testing for a possible outlier influence of the included studies in the results ${ }^{91}$ showed that no study differed significantly from the rest of the data (Table 7). In terms of publication bias, the funnel plot (Figure 5) showed no asymmetry according to both Egger's regression test $(z=-1.21$; $p=0.23$ ) and the rank correlation analysis (Kendall's tau = $-0.29 ; p=0.29$ ).

\section{General anxiety indexes}

Along with the specific anxiety measures, 9 of the 11 included studies reported pre/post general BAI and HAM-A scores for the stimulation and sham groups, we ran a separate meta-analysis for these scales. Similar to the specific 


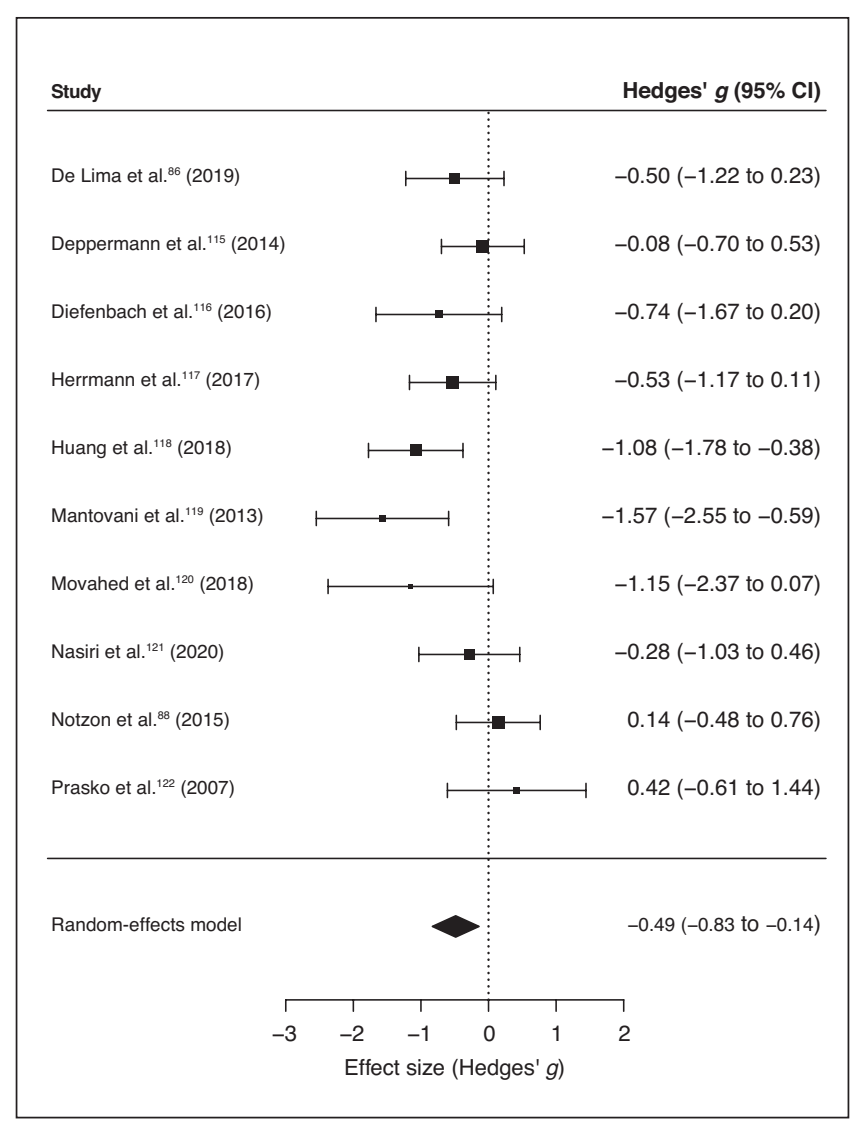

Figure 3: Forest plot of the effect size of noninvasive brain stimulation on continuous specific anxiety questionnaire scores. $\mathrm{Cl}=$ confidence interval. anxiety symptoms, the random effect model for general anxiety indexes showed a significant medium to large effect of noninvasive brain stimulation on the reduction of general anxiety scores compared to sham treatment (overall SMD $-0.81,95 \%$ CI -1.45 to -0.18 ; $p=0.012$; see Table 7 for complete results and Figure 6 for a forest plot).

For specific symptoms, $I^{2}$ and $Q$ statistics suggested high heterogeneity across studies, and a Baujat plot suggested that study $3^{87}$ was the main source of variance (Figure 7 ). Indeed, the influence test highlighted this study as an outlier (Table 7). Therefore, we re-ran the random-effects model excluding this study from the pool, and the global effect of noninvasive brain stimulation on the reduction of general anxiety scores remained significant (overall SMD $-0.57,95 \% \mathrm{CI}$ -1.06 to $-0.07 ; p=0.024$; see Table 7 for complete results). No other study was a significant outlier. Therefore we proceeded with the moderation analysis using the original set of 9 studies. The inclusion of moderators in the model was not significant ( $p>0.19$, Table 8). Funnel plot asymmetry (Figure 8) was nonsignificant for Egger's regression test $(z=-0.31, p=0.76)$ and rank correlation analysis (Kendall's tau $=-0.17 ; p=0.61$ ).

\section{Depression scales}

Seven of the final pool studies reported depression scale scores (Table 1) before and after the intervention. The random-effects model reported a significant global effect of noninvasive brain stimulation in reducing the scores on the depression inventories compared to sham interventions (overall SMD -0.98 , $95 \%$ CI -1.62 to $-0.35 ; p=0.002$; see Table 7 for the complete results and Figure 9 for a forest plot).

$I^{2}$ and $Q$ statistics suggested high heterogeneity across studies. The Baujat plot (Figure 10) suggested that study $2^{87}$

Table 8: Results of the moderation analysis for specific and general anxiety scores and depression scores

\begin{tabular}{|c|c|c|c|c|}
\hline Moderator & SMD (95\% Cl) & $z$ & $p$ & $Q_{1}$ \\
\hline \multicolumn{5}{|c|}{ Specific anxiety measure } \\
\hline Session number & $-0.0414(-0.1038$ to 0.0209$)$ & -1.3019 & 0.19 & 1.6950 \\
\hline Technique & $-0.2827(-0.7443$ to 0.1788$)$ & -1.2006 & 0.23 & 1.4415 \\
\hline Target region & $-0.4963(-1.2778$ to 0.2852$)$ & -1.2447 & 0.21 & 1.5493 \\
\hline Protocol type & $-0.4965(-1.1366$ to 0.1435$)$ & -1.5205 & 0.13 & 2.3118 \\
\hline \multicolumn{5}{|c|}{ General anxiety measure } \\
\hline Session number & $-0.0723(-0.1811$ to 0.0364$)$ & -1.3039 & 0.19 & 1.7001 \\
\hline Technique & $-0.1830(-1.2449$ to 0.8790$)$ & -0.3377 & 0.74 & 0.1140 \\
\hline Target region & $-0.8212(-2.2992$ to 0.6568$)$ & -1.0890 & 0.28 & 1.1858 \\
\hline Protocol type & $0.2243(-1.2106$ to 1.6592$)$ & 0.3064 & 0.76 & 0.0939 \\
\hline \multicolumn{5}{|l|}{ Depression measure } \\
\hline Session number & $-0.0777(-0.1634$ to 0.0080$)$ & -1.7760 & $0.076^{\star}$ & 3.1542 \\
\hline Technique & $0.5794(-0.7260$ to 1.8847$)$ & 0.8699 & 0.38 & 0.7567 \\
\hline Target region & $-0.6709(-2.9417$ to 1.5998$)$ & -0.5791 & 0.56 & 0.3354 \\
\hline Protocol type & $0.8540(-0.5639$ to 2.2718$)$ & 1.1805 & 0.24 & 1.3935 \\
\hline Comorbidity & $0.9563(-0.3677$ to 2.2803$)$ & 1.4157 & 0.16 & 2.0042 \\
\hline
\end{tabular}

$\mathrm{Cl}=$ confidence interval; dIPFC = dorsolateral prefrontal cortex; iTBS = intermittent theta burst stimulation; rTMS = repetitive transcranial magnetic stimulation; $S M D=$ standardized mean difference (effect size); tDCS = transcranial direct current stimulation. The applied technique (iTBS, rTMS, tDCS), target region (left vs. right dIPFC) and protocol type (excitatory v. inhibitory) moderators were categorical variables; session number was a numerical variable. For the depression outcome measure only, we computed whether the presence of comorbid depression influenced the outcome of the scores. $z=z$ score associated with the SMD value; $p=p$ value associated with the $z$ score in the same row. ${ }^{*} p<0.10$. 


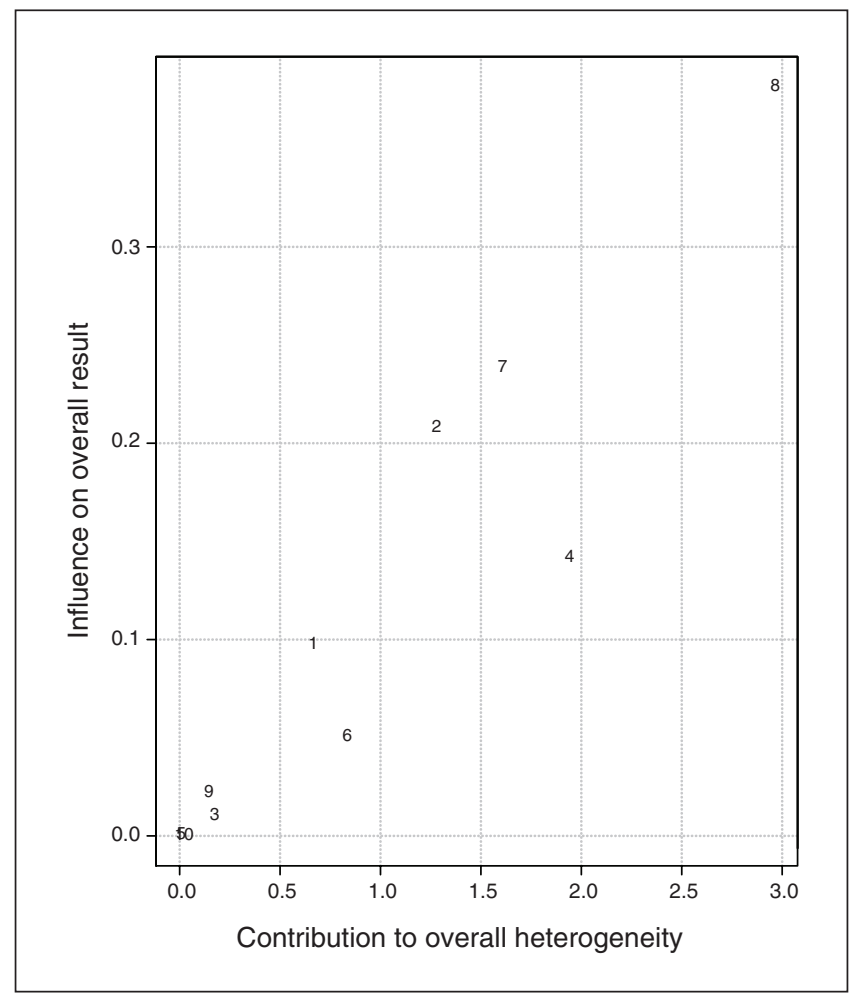

Figure 4: Baujat plot of study distribution in terms of heterogeneity for continuous specific anxiety questionnaire scores. On visual inspection, study $8^{119}$ seemed to contribute most to the statistical heterogeneity of the included studies.

was the main source of variance. The influence test identified this study as an outlier (Table 7). Still, even excluding this study from the meta-analysis the model highlighted a significant effect of noninvasive brain stimulation on reduction of depression scores (overall SMD $-0.64,95 \%$ CI -0.98 to -0.31 ; $p<0.001$; see Table 7 for complete results). No further study resulted in an outlier from the influence analysis. Therefore, we proceeded with the moderation analysis using the original set of 7 studies. Analysis of moderators indicated a trend toward significance for the number of stimulation sessions on the reduction of depression symptoms $\left(\mathrm{QM}_{1}=3.1\right.$, $p=0.08)$, with a higher reduction when the number of sessions increased. We found no effect for the presence of comorbidity in depression scores after treatment $(p=0.16)$. Funnel plot asymmetry (Figure 11) was nonsignificant for both the Egger's regression test $(z=-0.8, p=0.43)$ and the rank correlation analysis (Kendall's tau $=-0.07 ; p>0.99$ ).

\section{Discussion}

\section{Rationale and description of the study procedure}

Over the last few decades, the high rate of nonresponders to conventional treatment and low adherence to pharmacological interventions because of significant adverse effects has led to increasing demand for novel and complementary treat-

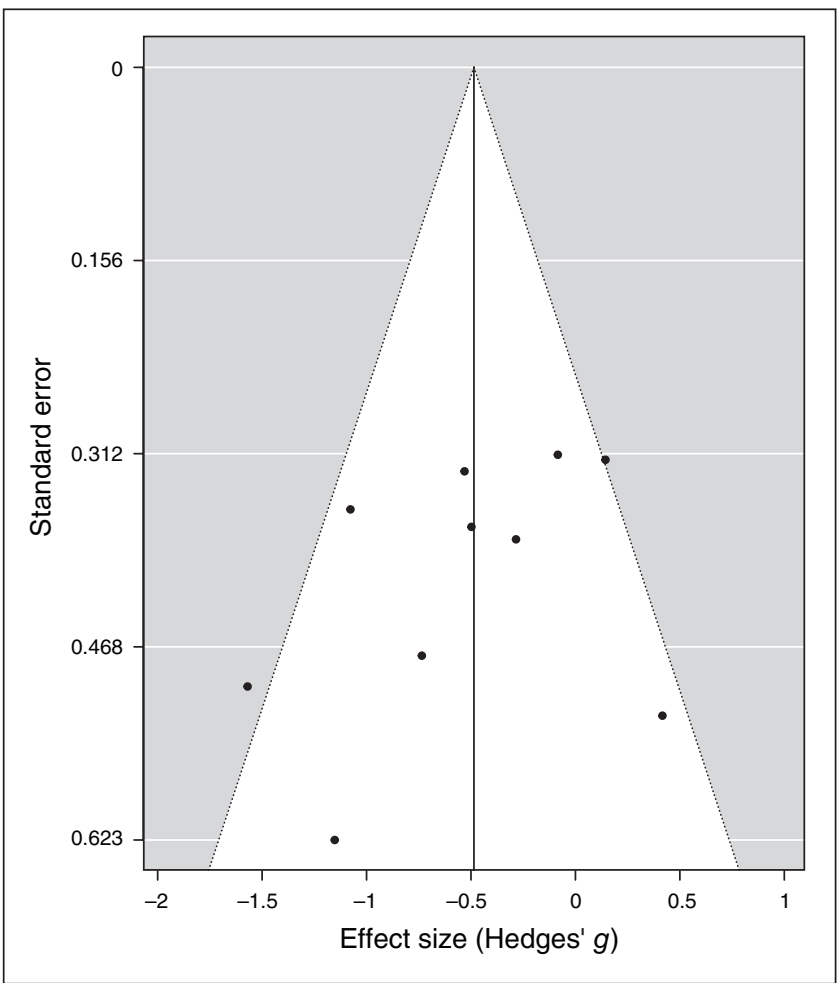

Figure 5: Publication bias assessed by funnel plot for continuous specific anxiety questionnaire scores.

ment approaches, including noninvasive brain stimulation. The effectiveness of rTMS in depression is well recognized and its clinical use is accepted worldwide; ${ }^{40}$ as well, recent expert guidelines for tDCS have pointed in the same direction. ${ }^{66}$ And yet, to date very little evidence has been available for the efficacy of noninvasive brain stimulation in anxiety disorders ${ }^{65-67,141}$ because of a low number of studies specifically investigating this topic.

We conducted a systematic review and quantitative analysis of the effectiveness of noninvasive brain stimulation in ameliorating the clinical symptoms of anxiety disorders. We included peer-reviewed original studies written in English in the present work. Given the importance of comparison with a placebo or control treatment, we included only studies that compared real stimulation with sham or control conditions.

Overall, 11 articles met our inclusion criteria. Studies differed in terms of the specific anxiety disorder they investigated: 10 of 11 studies (all but Dilkov and colleagues ${ }^{87}$ ) reported using disorder-specific questionnaires (e.g., the Panic Disorder Severity Scale in panic disorder). As well, 9 of 11 studies (all but Notzon and colleagues ${ }^{88}$ and Diefenbach and colleagues ${ }^{116}$ ) included a general anxiety measure (HAM-A or BAI). Therefore, we ran 2 separate meta-analyses for anxiety symptoms. The first included the results from a specific disorder questionnaire used in each study. The second included the results from a general anxiety questionnaire (HAM-A or BAI; the clinician-administered HAM-A was preferred when available). Finally, 7 studies $^{86,87,116,118-121}$ also included scores on a depression scale (HAM-D or Beck 


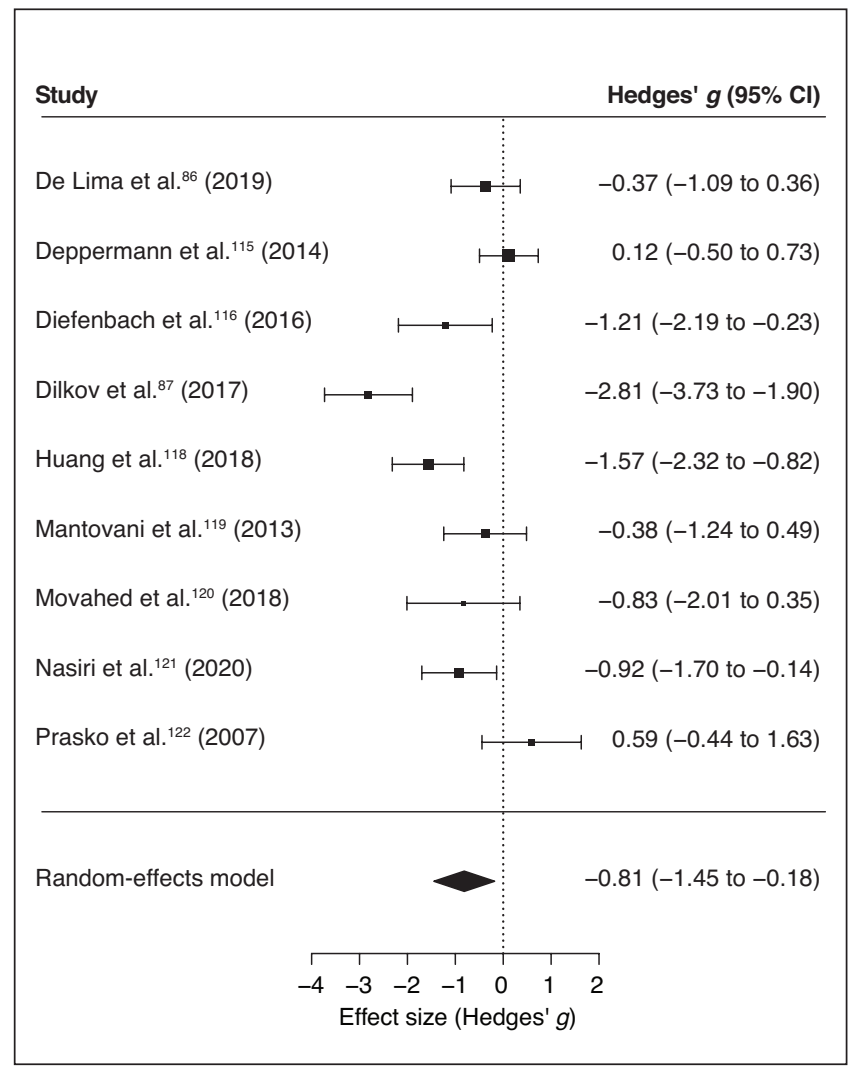

Figure 6: Forest plot of the effect size of noninvasive brain stimulation on continuous general anxiety questionnaire scores. $\mathrm{Cl}=$ confidence interval.

Depression Inventory), the focus of our third meta-analysis. It is well known that anxiety and depression are often comorbid and share some commonalities in the neural substrate involved. For this reason, we wanted to investigate whether noninvasive brain stimulation was useful in reducing symptoms of depression as well as anxiety.

\section{Main effect of noninvasive brain stimulation on anxiety and moderation analysis}

Our findings highlighted a significant medium effect of stimulation in decreasing anxiety scores compared to control conditions, suggesting that noninvasive brain stimulation can be useful in reducing symptoms of anxiety in patients. This effect was significant for both the disorder-specific and general anxiety measures, in line with the high correlation found between the 2 measures of anxiety (0.6), and might have been because of changes in symptoms that are shared by the various anxiety disorders. Crucially, the effect was not likely to have been influenced by publication or reporting bias. In line with previous systematic reviews ${ }^{35}$ and meta-analyses, ${ }^{72,73}$ we also acknowledge the limitations of these results, which are based on a restricted sample of studies but a relatively large pool of patients (318 in total).

We included only representative moderators in our moderation analyses because of the small number of studies in our

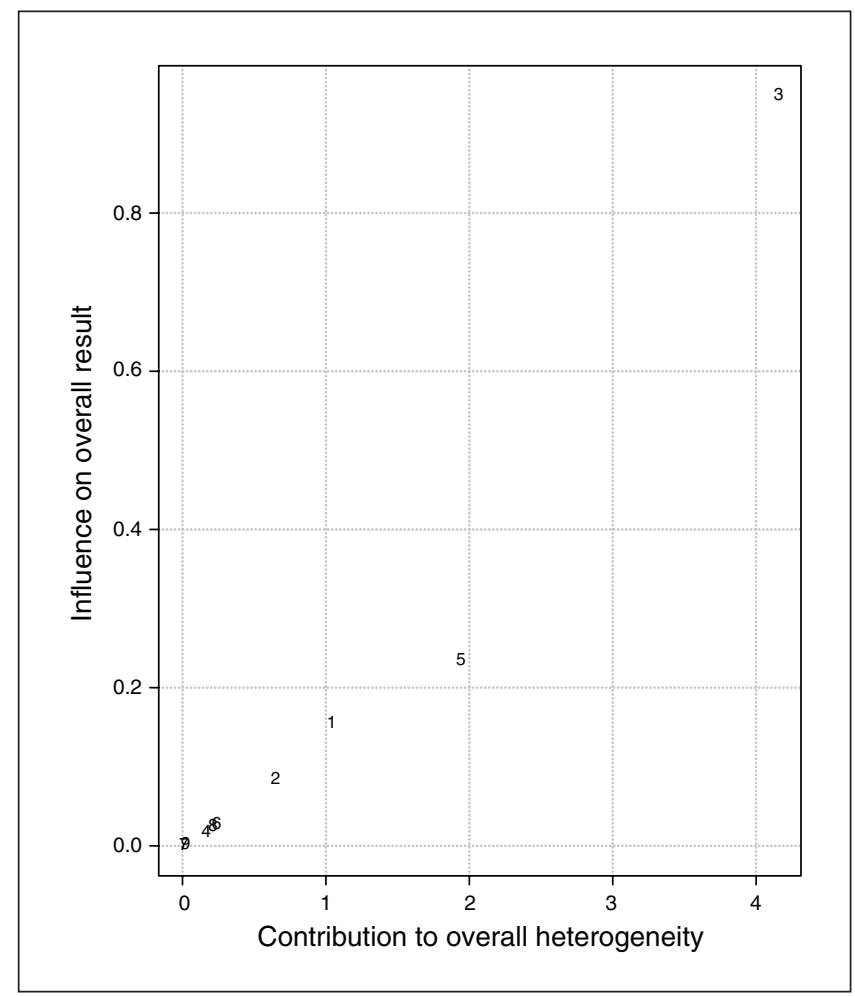

Figure 7: Baujat plot of study distribution in terms of heterogeneity for continuous general anxiety questionnaire scores. On visual inspection, study $3^{87}$ seemed to contribute most to the statistical heterogeneity of the included studies.

analysis. For example, only 2 studies targeted the right parietal region $\mathrm{PPC}^{118}$ and the ventromedial prefrontal cortex, ${ }^{117}$ all of the others targeted the dIPFC. Therefore, we ran the moderation analysis comparing stimulation of the left versus the right dIPFC. The analysis of moderators did not highlight any significant predictors, possibly because of the limited number of available studies. Only the number of stimulation sessions revealed a trend toward significance: depressive symptoms decreased for studies that included more sessions, in line with another recent meta-analysis. ${ }^{142}$ The influence of number of sessions in modulating depressive symptoms is debated but still controversial. Some studies and meta-analyses have reported a nonsignificant effect of dosage on symptom modulation, ${ }^{143,144}$ and others have suggested that at least $20-30$ sessions (or more) are required for optimal effects. ${ }^{145,146}$

$Q$ statistics and $I^{2}$ suggested high heterogeneity across studies, probably because of methodological differences across the selected works. Indeed, protocols varied with respect to participant diagnosis and treatment, the inclusion of associated therapies and protocol-specific parameters, target brain regions and the duration of the intervention. Specifically, participant diagnosis included generalized anxiety disorder (combined with insomnia or major depression), panic disorder (with or without agoraphobia and sometimes with comorbid major depression) and specific spider phobia. There was also heterogeneity in participants' ability to combine noninvasive brain stimulation with a medication or 


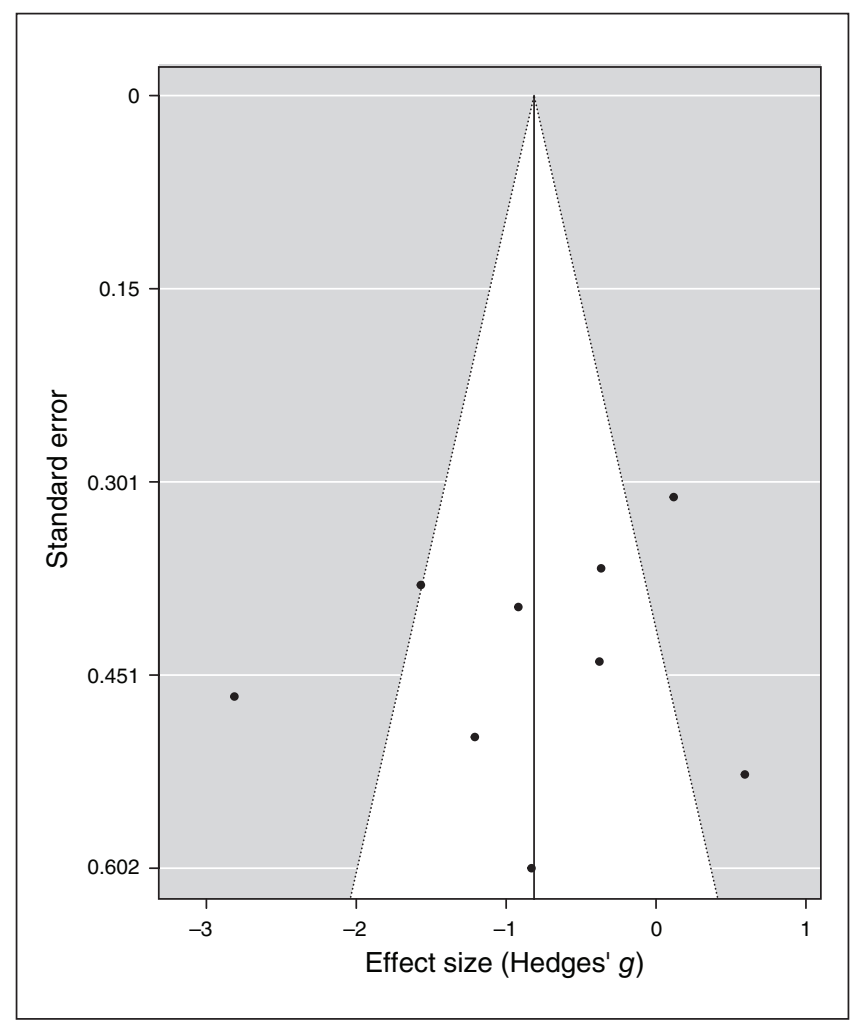

Figure 8: Publication bias assessed by funnel plot for continuous general anxiety questionnaire scores.

psychological treatment. In 3 studies ${ }^{88,120,121}$ participants were not allowed to follow a drug therapy; in the other, they could continue their treatment or follow a new one. ${ }^{115}$ In the latter situation, a time interval was established before starting noninvasive brain stimulation treatment; the interval varied across studies but was at least 3 weeks. Only 4 protocols $88,115,117,121$ included a psychological intervention.

\section{Combination of psychological intervention and neurostimulation}

Psychological and stimulation interventions were not always combined in the same sessions; in other words, they were not sequentially or simultaneously time-locked. ${ }^{41}$ Indeed, in 1 study, ${ }^{115}$ participants took part in 3 group sessions of panic disorder psychoeducation, separate from the noninvasive brain stimulation. In another study, ${ }^{121}$ tDCS was applied in the last 2 weeks of an emotional disorder psychological treatment process (unified protocol), but the authors did not specify whether tDCS was applied before, during or after treatment. Only Notzon and colleagues ${ }^{88}$ and Herrmann and colleagues ${ }^{117}$ provided a combined approach to a specific phobia, delivering iTBS before virtual reality exposure. Notzon and colleagues ${ }^{88}$ did not report any changes as a result of the single-session intervention, but in the study by Herrmann and colleagues, ${ }^{117}$ the 2 -session treatment led to a reduction in anxiety symptom scores. As previously highlighted by other researchers (see Sathappan and colleagues ${ }^{41}$

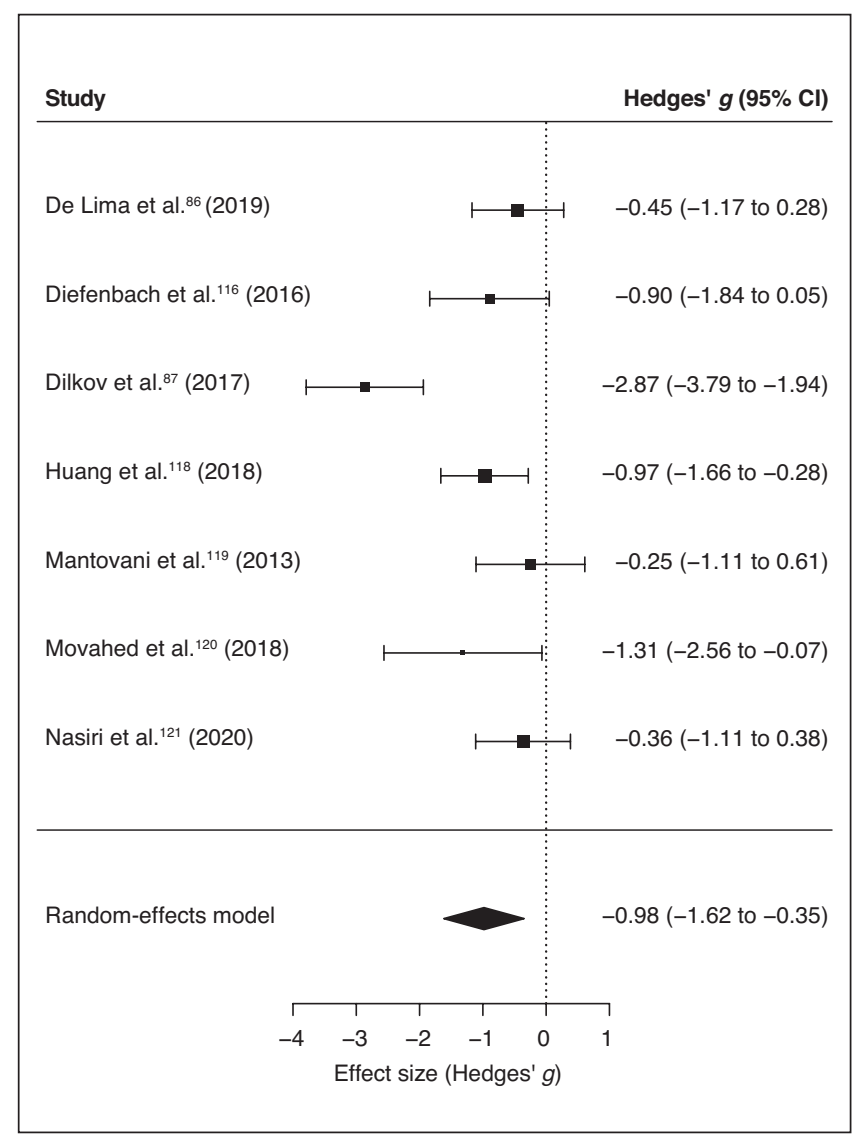

Figure 9: Forest plot of the effect size of noninvasive brain stimulation on continuous depression questionnaire scores. $\mathrm{Cl}=$ confidence interval.

for a recent review), the effect of combining behavioural or cognitive interventions with noninvasive brain stimulation is a gap in neuropsychiatric literature research. Indeed, it is well known that the effects of noninvasive brain stimulation are state dependent, meaning that the state of the stimulated regions during stimulation has a great influence on its effects on cortical excitability ${ }^{57,147-149}$ and behaviour. ${ }^{150-152}$ Moreover, converging evidence has suggested that both stimulation and psychotherapy can modulate brain connectivity, ${ }^{153,154}$ pointing to the possible importance of time-locking brain stimulation and behavioural engagement to investigate the possibility of maximizing their effects. A similar approach has been applied with stroke patients in the neurorehabilitation field, combining noninvasive brain stimulation with motor and speech training (for recent reviews, see Breining and Sebastian ${ }^{155}$ and Pruski and Cantarero ${ }^{156}$ ). In neuropsychiatric disorders, the investigation of combined interventions is still in its infancy, ${ }^{41,157}$ even for the treatment of depression, which has received more research attention..$^{41,144}$ In anxiety disorders, Heeren and colleagues ${ }^{113}$ combined the attentional bias modification technique with anodal and sham tDCS to reduce the bias for threat in patients with social anxiety. The study had a crossover design; participants performed only 2 sessions - a sham one and a real one - and the authors 


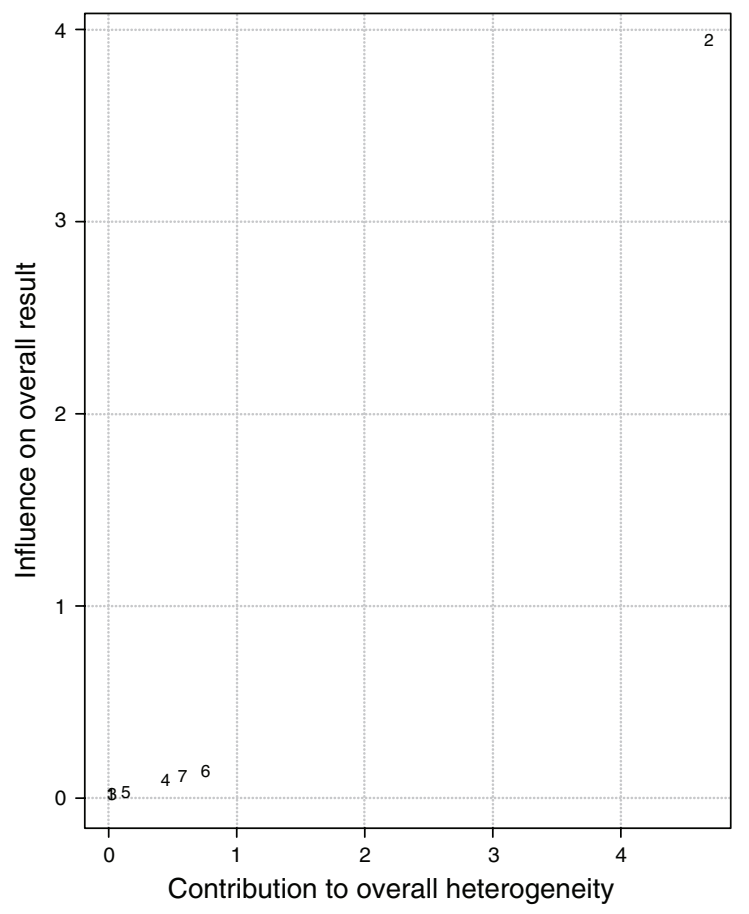

Figure 10: Baujat plot of study distribution in terms of heterogeneity for continuous depression questionnaire scores. On visual inspection, study $2^{87}$ seemed to contribute most to the statistical heterogeneity of the included studies.

reported a significant reduction in bias in the real stimulation condition compared to the sham stimulation. Segrave and colleagues $^{158}$ combined tDCS with simultaneous cognitive treatment (cognitive control therapy) in patients with MDD in 5 consecutive daily sessions. Real and sham tDCS led to equal improvements in depression symptoms after the fifth session of the protocol; however, effects were maintained at 3-week follow-up in only the group assigned to the real stimulation. In patients with schizophrenia, brain stimulation has been combined with cognitive remediation in an attempt to improve the cognitive deficits typical of the disease, but this has produced mixed results. ${ }^{159}$ There is evidence from experimental, behavioural and clinical research suggesting that the coupling of noninvasive brain stimulation with a concomitant treatment might enhance efficacy compared to each intervention alone. However, results are scarce and controversial, and this topic needs further investigation.

\section{Noninvasive brain stimulation to treat anxiety}

Most of the noninvasive brain stimulation studies in our review included a TMS intervention - either rTMS $^{87,116-119,122}$ or iTBS. ${ }^{88,115}$ Only 3 studies ${ }^{86,120,121}$ used a tDCS intervention. This choice was in line with knowledge about the treatment of depression, in which rTMS is considered a useful method for treating drug-resistant depression and because rTMS has stronger spatial resolution than $\mathrm{tDCS} .{ }^{160}$ However, in a

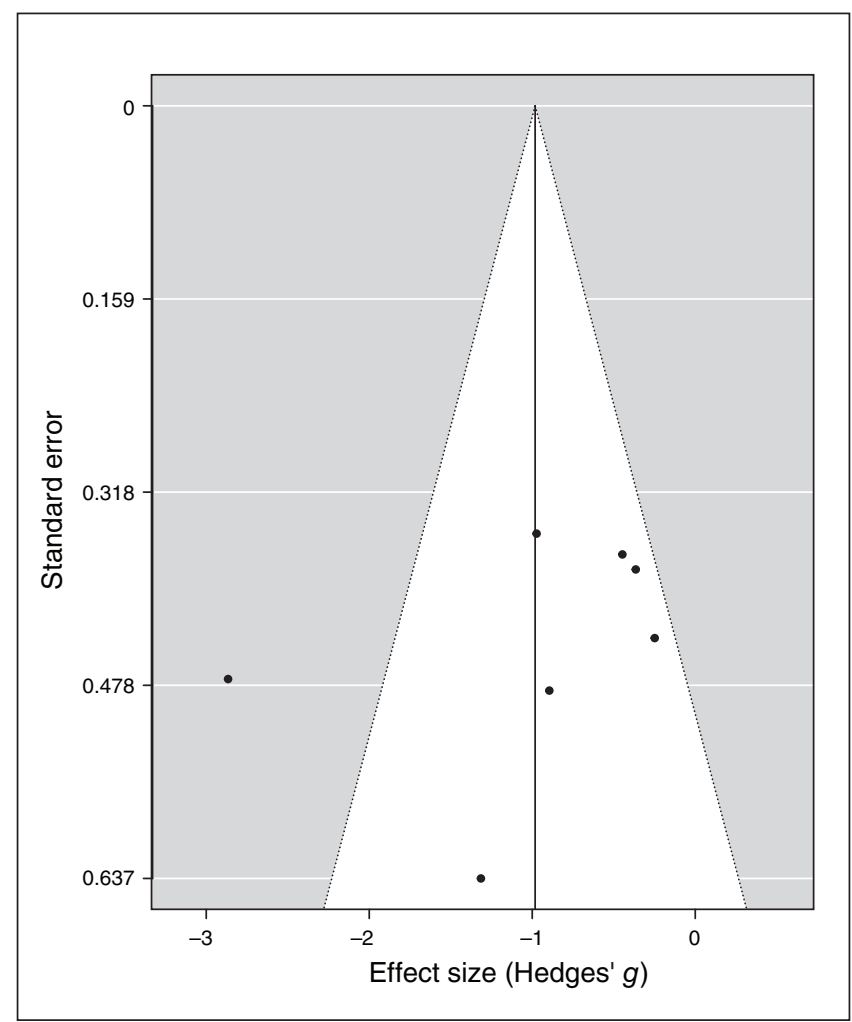

Figure 11: Publication bias assessed by funnel plot for continuous depression questionnaire scores.

combined approach, tDCS can be a convenient option, with fewer exogenous distractions related to rTMS-induced noise and fewer muscular contractions. The latter can be annoying or painful, especially when electrodes are applied to the prefrontal regions, the regions typically targeted in treatments we reviewed.

Recently, in addition to tDCS and rTMS, deep TMS has gained ground in treating the symptoms of obsessivecompulsive disorder ${ }^{161,162}$ and MDD (see Gellersen and Kedzior ${ }^{163}$ for a meta-analysis), and it has received US Food and Drug Administration clearance for both treatments. This technique uses the principles of TMS but delivers current through a specially designed $\mathrm{H}$-coil that can modulate cortical excitability up to $6 \mathrm{~cm}$ in depth, reaching not only cerebral cortex activity but also the activity of deeper neural circuits. ${ }^{164}$ To our knowledge, no previous studies have investigated deep TMS for anxiety disorders, and no articles about this technique appeared in our literature search combining "transcranial magnetic stimulation" or "TMS" with the 5 anxiety categories. However, given that we did not systematically search the term "deep TMS," we combined the key words "deep TMS" with each of the anxiety disorders in the 3 previously investigated databases. PubMed and Scopus research reported no results, and Web of Science search produced 3 results (the 3 results came from the combination of "deep TMS" and "generalized anxiety disorder"; 165 "deep TMS" and "specific phobia"; $; 66$ and "deep TMS" and "social anxiety disorder"167): a nonoriginal 
study, ${ }^{165}$ a study in animals ${ }^{166}$ and a study in patients with depression. ${ }^{167}$ The lack of studies evaluating deep TMS for anxiety disorders reflects the general limited number of studies investigating noninvasive brain stimulation and anxiety disorders compared to other psychiatric conditions and points to the importance of shedding light in this field.

\section{Comorbidity of anxiety and depression}

With respect to the clinical comorbidity of anxiety and depressive disorders, our results highlighted the efficacy of noninvasive brain stimulation in reducing depression scores compared to the control condition, an effect that was not merely shown in studies in which comorbidity was formally diagnosed in the sample population. This finding was in line with previous studies that investigated the effectiveness of rTMS in reducing anxiety symptoms during the treatment of patients with depression. In one of the largest studies, Chen and colleagues ${ }^{168}$ investigated the efficacy of left-dlPFC high-frequency, right-dlPFC lowfrequency and sequential bilateral rTMS (i.e., highfrequency left dlPFC followed by low-frequency right dlPFC) in a sample of 697 participants. The stimulation protocols showed the overall efficacy of the 3 protocols in reducing anxiety and depressive symptoms without indicating that one protocol had a stronger therapeutic effectiveness over the other. In another study, Clarke and colleagues ${ }^{103}$ analyzed data from a sample of 248 patients with treatment-resistant depression, of whom 172 had 1 or more comorbid anxiety disorders. rTMS was applied using $1 \mathrm{~Hz}$ to the right dlPFC or a sequential bilateral protocol (10 Hz over the left dlPFC and $1 \mathrm{~Hz}$ over the right dlPFC). Interestingly, rTMS reduced anxiety levels in patients with and without a formal anxiety diagnosis, as shown by a significant reduction in HAM-A scores in both subgroups. Similarly, in our sample 9 of 11 interventions targeted the left or right dlPFC; only 2 studies ${ }^{117,118}$ targeted a different site, namely the right posterior parietal cortex and the ventromedial prefrontal cortex, respectively. Crucially, when applied over the right hemisphere (dlPFC or posterior parietal cortex) stimulation was inhibitory (except for Dilkov and colleagues, ${ }^{87}$ who applied an excitatory protocol over the right dlPFC), with cathodal tDCS or low-frequency $(1 \mathrm{~Hz})$ rTMS. Over the left dlPFC, all studies applied excitatory protocols as iTBS and anodal tDCS. This choice was in line with previous knowledge related to the neural underpinning of anxiety disorders, which suggests that the left dlPFC is typically hypoactive in anxiety disorders, and the right dIPFC seems hyperactive. ${ }^{33,34,169}$ The overlap between the targeted regions and inhibition or excitation protocols explains the reported efficacy of noninvasive brain stimulation in reducing both anxiety and depression scores compared to control conditions. Indeed, although international guidelines and the US Food and Drug Administration approval recommend the application of excitatory (high-frequency rTMS, deep TMS or anodal tDCS) stimulation over the left dlPFC, it is known that noninvasive brain stimulation can also influence brain excitability through interhemispheric projections. Based on this idea, a change in excitability in one hemisphere - also induced by exogenous stimulation such as noninvasive brain stimulation - might induce indirect changes in the excitability of the other hemisphere, and eventually in behavioural outcomes. Such an effect has been reported for cognitive and motor tasks involving the prefrontal and frontal regions ${ }^{170-172}$ and for neurorehabilitation, especially involving post-stroke patients. ${ }^{173,174}$

The latter result is exciting and paves the way to specifically investigating the phenomenological overlapping of depression and anxiety disorders. Indeed, the fact that the stimulation of a similar brain network modulates both anxiety and depression symptoms, and some antidepressant drugs do the same (serotonin/adrenaline reuptake inhibitors show an effectiveness in treating both disorders) suggests a similarity in the neurochemical basis of the 2 syndromes. A recent study by Maggioni and colleagues ${ }^{175}$ specifically investigated neural commonalities and differences between anxiety and depression using structural MRI. Although this study was preliminary, its findings suggested that the clinical similarities between major depression and anxiety might rely on common prefrontal alterations involving left orbitofrontal thinning, while frontotemporal abnormalities are traceable in MDD and parietal abnormalities are specific to panic and social anxiety disorders.

It is interesting to note that the prefrontal regions are generally linked to emotional processing and regulation, ${ }^{31,176-178}$ which are known to be at the basis of the development and maintenance of anxiety and depression. For instance, studies in healthy participants have suggested that stimulation of the left dlPFC has positive effects on modulating several cognitive, emotional and neural processes that are relevant to anxiety. ${ }^{29,113,179,180}$ (See Stein and colleagues ${ }^{181}$ for a systematic review of the effects of tDCS in anxiety disorders or anxious behaviours in healthy participants.)

A final comment should be made about the outcome measures. The included studies used scores on validated questionnaires as outcome measures. However, only 1 study $^{88}$ investigated psychophysiological measures in addition to questionnaire results, evaluating skin conductance level and heart rate variability. The authors found no differences in skin conductance level but they did find a modulation in heart rate variability in the iTBS group versus the sham group, independent of the participant sample (patients v. healthy individuals). It is not usual to measure implicit psychophysiological measures as indicators of treatment effectiveness when applying noninvasive brain stimulation. ${ }^{35}$ However, such measures might be an index not only for assessing treatment improvement, but also for dosing the intervention in a flexible way and as predictors of treatment outcomes. For example, in a previous study with veterans affected by posttraumatic stress disorder, the baseline startle response to virtual reality combatrelated scenes was predictive of clinical outcomes: higher startle responses predicted greater changes in symptom severity at the end of the 6 weeks of treatment. ${ }^{182}$ 


\section{Limitations}

Research investigating the relationship between noninvasive brain stimulation and anxiety disorders is still in an embryonic state. Overall, just a few studies targeting patients with anxiety disorders are available; many authors have focused instead on healthy participants with a high trait of anxiety. For the studies that did include a clinical sample, only a few protocols investigated the efficacy of noninvasive brain stimulation at a group level. Moreover, the inclusion of a sham or control condition for comparison with the stimulation condition is not standard in research, despite the fact that the placebo effect of noninvasive brain stimulation techniques is well-known in both participants and experimenters, highlighting the importance of applying double-blind procedures. ${ }^{183,184}$ Future studies should also move in the direction of coupling noninvasive brain stimulation with behavioural or cognitive interventions, investigating whether combined treatment is more effective than monotherapy. Another crucial point about the efficacy of noninvasive brain stimulation protocols is based on sex. A limitation of the present study was the lack of a regression analysis including sex as a moderator. Unfortunately, none of the included studies reported outcome scores separately based on participants' sex.

\section{Conclusion}

Although our findings are preliminary, they suggest that noninvasive brain stimulation can be effective in decreasing anxiety and depressive symptoms in anxiety disorders, paving the way for treatment protocols that include noninvasive brain stimulation. Further research is needed to optimize the protocols in terms of duration, location, intensity and technique, and to define potential interindividual differences in response to neuromodulation induced by noninvasive brain stimulation..$^{55}$

Affiliations: From the Department of Psychology, University of Milano Bicocca, Milan, Italy (Vergallito, Pisoni, Punzi, Romero Lauro); the Neuromi, Milan, Italy (Vergallito, Gallucci, Pisoni, Romero Lauro); the Department of Neuroscience, School of Medicine and Surgery, University of Milano-Bicocca, Monza, Italy (Gallucci); the Studi Cognitivi, Milan, Italy (Caselli, Ruggiero, Sassaroli); and the Faculty of Psychology, Sigmund Freud University, Milan, Italy (Caseli, Ruggiero, Sassaroli).

Funding: A. Vergallito was supported in part by a 2019 NARSAD Young Investigator Grant, Brain \& Behavior Research Foundation.

\section{Competing interests: None declared.}

Contributors: A. Vergallito, A. Galluci, A. Pisoni and L. Romero Lauro designed the study. A. Vergallito, A. Gallucci, A. Pisoni and M. Punzi acquired and analyzed the data, which G. Caselli, G. Ruggiero, S. Sassaroli and L. Romero Lauro interpreted. A. Vergallito, A. Galluci, A. Pisoni and M. Punzi wrote the article, which G. Caselli, G. Ruggiero, S. Sassaroli and L. Romero Lauro reviewed. All authors approved the final version to be published and can certify that no other individuals not listed as authors have made substantial contributions to the paper.

Content licence: This is an Open Access article distributed in accordance with the terms of the Creative Commons Attribution (CC BY-NC-ND 4.0) licence, which permits use, distribution and reproduction in any medium, provided that the original publication is properly cited, the use is noncommercial (i.e., research or educational use), and no modifications or adaptations are made. See: https:/ / creativecommons.org/licenses/by-nc-nd/4.0/

\section{References}

1. James SL, Abate D, Hassen Abate K, et al. Global, regional, and national incidence, prevalence, and years lived with disability for 354 diseases and injuries for 195 countries and territories, 1990-2017: a systematic analysis for the Global Burden of Disease Study 2017. Lancet 2018;392:1789-858.

2. Wittchen H-U, Jacobi F, Rehm J, et al. The size and burden of mental disorders and other disorders of the brain in Europe 2010. Eur Neuropsychopharmacol 2011;21:655-79.

3. GBD 2015 Risk Factors Collaborators. Global, regional, and national comparative risk assessment of 79 behavioural, environmental and occupational, and metabolic risks or clusters of risks, 19902015: a systematic analysis for the Global Burden of Disease Study 2015. Lancet 2016;388:1659.

4. Craske MG, Stein MB, Eley TC, et al. Correction: anxiety disorders. Nat Rev Dis Primers 2017;3:1.

5. Lijster, JM, Dierckx B, Utens EMWJ, et al. The age of onset of anxiety disorders: a meta-analysis. Can J Psychiatry 2017;62:237-46.

6. Beesdo K, Knappe S, Pine DS. Anxiety and anxiety disorders in children and adolescents: developmental issues and implications for DSM-V. Psychiatr Clin North Am 2009;32:483-524.

7. American Psychiatric Association. Diagnostic and statistical manual of mental disorders. Fifth edition. Arlington (VA): American Psychiatric Association Publishing 2013.

8. International statistical classification of diseases and related health problems. 10th rev. Geneva: World Health Organization; 1993.

9. Schmidt CK, Khalid S, Loukas M, et al. Neuroanatomy of anxiety: a brief review. Cureus 2018;10:e2055.

10. Bas-Hoogendam JM, Groenewold NA, Aghajani M, et al. ENIGMA-anxiety working group: rationale for and organization of large-scale neuroimaging studies of anxiety disorders. Hum Brain Mapp 2020 [Epub ahead of print]. doi: 10.1002/hbm.25100.

11. Duval ER, Javanbakht A, Liberzon I. Neural circuits in anxiety and stress disorders: a focused review. Ther Clin Risk Manag 2015;11:115.

12. Holzschneider K, Mulert C. Neuroimaging in anxiety disorders. Dialogues Clin Neurosci 2011;13:453.

13. Taylor JM, Whalen PJ. Neuroimaging and anxiety: the neural substrates of pathological and non-pathological anxiety. Curr Psychiatry Rep 2015;17:49.

14. Furmark T, Tillfors M, Garpenstrand H, et al. Serotonin transporter polymorphism related to amygdala excitability and symptom severity in patients with social phobia. Neurosci Lett 2004;362:189-92.

15. Lorberbaum JP, Kose S, Johnson MR, et al. Neural correlates of speech anticipatory anxiety in generalized social phobia. Neuroreport 2004;15:2701-5.

16. Tillfors M, Furmark T, Marteinsdottir I, et al. Cerebral blood flow in subjects with social phobia during stressful speaking tasks: a PET study. Am J Psychiatry 2001;158:1220-6.

17. Schneider F, Weiss U, Kessler C, et al. Subcortical correlates of differential classical conditioning of aversive emotional reactions in social phobia. Biol Psychiatry 1999;45:863-71.

18. Markovic V, Vicario CM, Yavari F, et al. A systematic review on the effect of transcranial direct current and magnetic stimulation on fear memory and extinction. Front Hum Neurosci 2021;15:655947.

19. Labuschagne I, Luan Phan K, Wood A, et al. Oxytocin attenuates amygdala reactivity to fear in generalized social anxiety disorder. Neuropsychopharmacology 2010;35:2403-13.

20. Shah SG, Klumpp H, Angstadt M, et al. Amygdala and insula response to emotional images in patients with generalized social anxiety disorder. J Psychiatry Neurosci 2009;34:296.

21. Ball TM, Sullivan S, Flagan T, et al. Selective effects of social anxiety, anxiety sensitivity, and negative affectivity on the neural bases of emotional face processing. Neuroimage 2012;59:1879-87.

22. Lipka J, Miltner WHR, Straube T. Vigilance for threat interacts with amygdala responses to subliminal threat cues in specific phobia. Biol Psychiatry 2011;70:472-8.

23. Gingnell M, Frick A, Engman J, et al. Combining escitalopram and cognitive-behavioural therapy for social anxiety disorder: randomised controlled fMRI trial. Br J Psychiatry 2016;209:229-35. 
24. Månsson KN, Carlbring P, Frick A, et al. Altered neural correlates of affective processing after internet-delivered cognitive behavior therapy for social anxiety disorder. Psychiatry Res 2013;214:229-37.

25. Phan KL, Coccaro EF, Angstadt M, et al. Corticolimbic brain reactivity to social signals of threat before and after sertraline treatment in generalized social phobia. Biol Psychiatry 2013;73:329-36.

26. Maren S, Holmes A. Stress and fear extinction. Neuropsychopharmacology 2016;41:58-79.

27. Maroun M, Kavushansky A, Holmes A, et al. Enhanced extinction of aversive memories by high-frequency stimulation of the rat infralimbic cortex. PLoS One 2012;7:e35853.

28. Etkin A, Wager TD. Functional neuroimaging of anxiety: a metaanalysis of emotional processing in PTSD, social anxiety disorder and specific phobia. Am J Psychiatry 2007;164:1476-88.

29. Ironside M, Browning M, Ansari TL, et al. Effect of prefrontal cortex stimulation on regulation of amygdala response to threat in individuals with trait anxiety: a randomized clinical trial. JAMA Psychiatry 2019;76:71-8.

30. Kim MJ, Loucks RA, Palmer AL, et al. The structural and functional connectivity of the amygdala: from normal emotion to pathological anxiety. Behav Brain Res 2011;223:403-10.

31. Motzkin JC, Philippi CL, Wolf RC, et al. Ventromedial prefrontal cortex is critical for the regulation of amygdala activity in humans. Biol Psychiatry 2015;77:276-84.

32. Kraus J, Frick A, Fischer H, et al. Amygdala reactivity and connectivity during social and non-social aversive stimulation in social anxiety disorder. Psychiatry Res Neuroimaging 2018;280:56-61.

33. Nitschke JB, Heller W. Distinguishing neural substrates of heterogeneity among anxiety disorders. Int Rev Neurobiol 2005;67:1-42.

34. Prasko J, Horácek J, Záleský R, et al. The change of regional brain metabolism (18FDG PET) in panic disorder during the treatment with cognitive behavioral therapy or antidepressants. Neuroendocrinol Lett 2004;25:340-8.

35. Vicario CM, Salehinejad MA, Felmingham K, et al. A systematic review on the therapeutic effectiveness of non-invasive brain stimulation for the treatment of anxiety disorders. Neurosci Biobehav Rev 2019;96:219-31.

36. Bandelow B, Lichte T, Rudolf S, et al. The German guidelines for the treatment of anxiety disorders. Eur Arch Psychiatry Clin Neurosci 2015;265:363-73

37. Katzman MA, Bleau P, Blier $P$, et al. Canadian clinical practice guidelines for the management of anxiety, posttraumatic stress and obsessive-compulsive disorders. BMC Psychiatry 2014;14:S1.

38. Fernandez E, Salem D, Swift JK, et al. Meta-analysis of dropout from cognitive behavioral therapy: magnitude, timing, and moderators. J Consult Clin Psychol 2015;83:1108.

39. Taylor S, Abramowitz JS, McKay D. Non-adherence and nonresponse in the treatment of anxiety disorders. J Anxiety Disord 2012; 26:583-9.

40. Brunoni AR, Sampaio-Junior B, Moffa AH, et al. Noninvasive brain stimulation in psychiatric disorders: a primer. Br J Psychiatry 2019;41: 70-81.

41. Sathappan AV, Luber BM, Lisanby SH. The dynamic duo: combining noninvasive brain stimulation with cognitive interventions. Prog Neuropsychopharmacol Biol Psychiatry 2019;89:347-60.

42. Wessel MJ, Zimerman M, Hummel FC. Non-invasive brain stimulation: an interventional tool for enhancing behavioral training after stroke. Front Hum Neurosci 2015;9:265.

43. Nitsche MA, Müller-Dahlhaus F, Paulus W, et al. The pharmacology of neuroplasticity induced by non-invasive brain stimulation: building models for the clinical use of CNS active drugs. J Physiol 2012;590:4641-62.

44. Kronberg G, Bridi M, Abel T, et al. Direct current stimulation modulates LTP and LTD: activity dependence and dendritic effects. Brain Stimul 2017;10:51-8.

45. Ziemann U. Thirty years of transcranial magnetic stimulation: where do we stand? Exp Brain Res 2017;235:973-84.

46. Reis J, Robertson E, Krakauer JW, et al. Consensus: can tDCS and TMS enhance motor learning and memory formation? Brain Stimul 2008;1:363.
47. Cirillo G, Pino GD, Capone F, et al. Neurobiological after-effects of non-invasive brain stimulation. Brain Stimul 2017;10:1-18.

48. Kobayashi M, Pascual-Leone A. Transcranial magnetic stimulation in neurology. Lancet Neurol 2003;2:145-56.

49. Fregni F, Pascual-Leone A. Technology insight: noninvasive brain stimulation in neurology - perspectives on the therapeutic potential of rTMS and tDCS. Nat Clin Pract Neurol 2007;3:383-93.

50. Nitsche MA, Paulus W. Excitability changes induced in the human motor cortex by weak transcranial direct current stimulation. J Physiol 2000;527:633

51. Brunoni AR, Nitsche MA, Bolognini N, et al. Clinical research with transcranial direct current stimulation (tDCS): challenges and future directions. Brain Stimul 2012;5:175-95.

52. Purpura DP, McMurtry JG. Intracellular activities and evoked potential changes during polarization of motor cortex. J Neurophysiol 1965;28:166-85.

53. Nitsche MA, Cohen LG, Wassermann EM, et al. Transcranial direct current stimulation: state of the art 2008. Brain Stimul 2008;1:206-23.

54. Castrillon G, Sollmann N, Kurcyus K, et al. The physiological effects of noninvasive brain stimulation fundamentally differ across the human cortex. Sci Adv 2020;6:eaay2739.

55. López-Alonso V, Cheeran B, Río-Rodríguez D, et al. Inter-individual variability in response to non-invasive brain stimulation paradigms. Brain Stimul 2014;7:372-80.

56. Pell GS, Roth Y, Zangen A. Modulation of cortical excitability induced by repetitive transcranial magnetic stimulation: influence of timing and geometrical parameters and underlying mechanisms. Prog Neurobiol 2011;93:59-98.

57. Pisoni A, Mattavelli G, Papagno C, et al. Cognitive enhancement induced by anodal tDCS drives circuit-specific cortical plasticity. Cereb Cortex 2018;28:1132-40.

58. Hui J, Tremblay S, Daskalakis ZJ. The current and future potential of transcranial magnetic stimulation with electroencephalography in psychiatry. Clin Pharmacol Ther 2019;106:734-46.

59. Kennedy NI, Lee WH, Frangou S. Efficacy of non-invasive brain stimulation on the symptom dimensions of schizophrenia: a metaanalysis of randomized controlled trials. Eur Psychiatry 2018;49: 69-77.

60. Kostova R, Cecere R, Thut G, et al. Targeting cognition in schizophrenia trough transcranial direct current stimulation: a systematic review and perspective. Schizophr Res 2020;220:300-10.

61. Trojak B, Sauvaget A, Fecteau S, et al. Outcome of non-invasive brain stimulation in substance use disorders: a review of randomized sham-controlled clinical trials. J Neuropsychiatry Clin Neurosci 2017;29:105-18.

62. Brunelin J, Mondino M, Bation R, et al. Transcranial direct current stimulation for obsessive-compulsive disorder: a systematic review. Brain Sci 2018;8:37.

63. Trevizol AP, Shiozawa P, Cook IA, et al. Transcranial magnetic stimulation for obsessive-compulsive disorder: an updated systematic review and meta-analysis. J ECT 2016;32:262-6.

64. Lefaucheur J-P, Aleman A, Baeken C, et al. Evidence-based guidelines on the therapeutic use of repetitive transcranial magnetic stimulation (rTMS). Clin Neurophysiol 2014;125:2150-206.

65. Lefaucheur J-P, Aleman A, Baeken C, et al. Evidence-based guidelines on the therapeutic use of repetitive transcranial magnetic stimulation (rTMS): an update (2014-2018). Clin Neurophysiol 2020;131:474-528.

66. Fregni F, El-Hagrassy MM, Pacheco-Barrios K, et al. Evidencebased guidelines and secondary meta-analysis for the use of transcranial direct current stimulation (tDCS) in neurological and psychiatric disorders. Int J Neuropsychopharmacol 2021;24:256-313.

67. Lefaucheur J-P, Aleman A, Baeken C, et al. Evidence-based guidelines on the therapeutic use of transcranial direct current stimulation (tDCS). Clin Neurophysiol 2017;128:56-92.

68. Iannone A, Cruz AP. de M., Brasil-Neto JP, et al. Transcranial magnetic stimulation and transcranial direct current stimulation appear to be safe neuromodulatory techniques useful in the treatment of anxiety disorders and other neuropsychiatric disorders. Arq Neuropsiquiatr 2016;74:829-35. 
69. Rodrigues PA, Luiza Zaninotto A, Neville LS, et al. Transcranial magnetic stimulation for the treatment of anxiety disorder. Neuropsychiatr Dis Treat 2019;15:2743.

70. Sagliano L, Atripaldi D, De Vita D, et al. Non-invasive brain stimulation in generalized anxiety disorder: a systematic review. Prog Neuropsychopharmacol Biol Psychiatry 2019;93:31-8.

71. Cirillo P, Gold AK, Nardi AE, et al. Transcranial magnetic stimulation in anxiety and trauma-related disorders: a systematic review and meta-analysis. Brain Behav 2019;9:e1284.

72. Cui, H. Jiang L, Wei $Y$, et al. Efficacy and safety of repetitive transcranial magnetic stimulation for generalised anxiety disorder: a meta-analysis. Gen Psychiatr 2019;32:e100051.

73. Trevizol, AP, Shiozawa P, Sato IA, et al. Transcranial magnetic stimulation for anxiety symptoms: an updated systematic review and meta-analysis. Abnorm Behav Psychol 2016;2:1.

74. Grimm S, Beck J, Schuepbach D, et al. Imbalance between left and right dorsolateral prefrontal cortex in major depression is linked to negative emotional judgment: an fMRI study in severe major depressive disorder. Biol Psychiatry 2008;63:369-76.

75. Hamilton JP, Etkin A, Furman DJ, et al. Functional neuroimaging of major depressive disorder: a meta-analysis and new integration of baseline activation and neural response data. Am J Psychiatry 2012;169:693-703.

76. Siegle GJ, Thompson W, Carter CS, et al. Increased amygdala and decreased dorsolateral prefrontal BOLD responses in unipolar depression: related and independent features. Biol Psychiatry 2007;61:198-209.

77. Beesdo K, Bittner A, Pine DS, et al. Incidence of social anxiety disorder and the consistent risk for secondary depression in the first three decades of life. Arch Gen Psychiatry 2007;64:903-12.

78. Bittner A, Goodwin RD, Wittchen HU, et al. What characteristics of primary anxiety disorders predict subsequent major depressive disorder? J Clin Psychiatry 2004;65:618-26.

79. Ressler KJ, Mayberg HS. Targeting abnormal neural circuits in mood and anxiety disorders: from the laboratory to the clinic. Nat Neurosci 2007;10:1116-24.

80. Choi KW, Kim Y-K, Jeon HJ. Comorbid anxiety and depression: clinical and conceptual consideration and transdiagnostic treatment. In: Kim Y-K, editor. Anxiety disorders: rethinking and understanding recent discoveries. Singapore: Springer Nature; 2020: 219-35.

81. Ionescu DF, Niciu MJ, Henter ID, et al. Defining anxious depression: a review of the literature. CNS Spectr 2013;18:252.

82. Moher D, Shamseer L, Clarke M, et al. Preferred reporting items for systematic review and meta-analysis protocols (PRISMA-P) 2015 statement. Syst Rev 2015;4:1.

83. Shamseer L, Moher D, Clarke M, et al. Preferred reporting items for systematic review and meta-analysis protocols (PRISMA-P) 2015: elaboration and explanation. BMJ 2015;349:g7647.

84. Ouzzani M, Hammady H, Fedorowicz Z, et al. Rayyan - a web and mobile app for systematic reviews. Syst Rev 2016;5:210.

85. Higgins JPT, Altman DG, Gøtzsche PC, et al. The Cochrane Collaboration's tool for assessing risk of bias in randomised trials. BMJ 2011;343:d5928.

86. de Lima AL, Azevedo Braga FM, Maciel Medeiros da Costa R, et al. Transcranial direct current stimulation for the treatment of generalized anxiety disorder: a randomized clinical trial. J Affect Disord 2019;259:31-7.

87. Dilkov D, Hawken ER, Kaludiev E, et al. Repetitive transcranial magnetic stimulation of the right dorsal lateral prefrontal cortex in the treatment of generalized anxiety disorder: a randomized, double-blind sham controlled clinical trial. Prog Neuropsychopharmacol Biol Psychiatry 2017;78:61-5.

88. Notzon S, Deppermann S, Fallgatter A, et al. Psychophysiological effects of an iTBS modulated virtual reality challenge including participants with spider phobia. Biol Psychol 2015;112:66-76.

89. Higgins JPT, Thomas J, Chandler J, et al, editors. Cochrane handbook for systematic reviews of interventions. 2nd ed. Chichester, United Kingdom: John Wiley \& Sons; 2019.

90. Follmann D, Elliott $P$, Suh IL, et al. Variance imputation for overviews of clinical trials with continuous response. J Clin Epidemiol 1992;45:769-73.
91. Viechtbauer W, Cheung MW. Outlier and influence diagnostics for meta-analysis. Res Synth Methods 2010;1:112-25.

92. Viechtbauer W. Conducting meta-analyses in R with the metafor package. J Stat Softw 2010;36:1-48.

93. Hedges LV. Distribution theory for Glass's estimator of effect size and related estimators. J Educ Stat 1981;6:107-28.

94. Field AP. Meta-analysis in clinical psychology research. In: Comer JS, Kendall PC, editors. Oxford handbook of research strategies for clinical psychology. New York: Oxford University Press; 2013: 317-35.

95. Higgins JPT, Thompson SG, Deeks JJ, et al. Measuring inconsistency in meta-analyses. BMJ 2003;327:557-60.

96. Del Re AC. A practical tutorial on conducting meta-analysis in R. Quant Methods Psychol 2015;11:37-50.

97. Kovalchik S. Tutorial on meta-analysis in R [tutorial]. R useR! conference; 2013 Jul 10-12; Albacete, Spain.

98. Polanin JR, Tanner-Smith EE, Hennessy EA. Estimating the difference between published and unpublished effect sizes: a meta-review. Rev Educ Res 2016;86:207-36.

99. Egger M, Smith GD, Schneider M, et al. Bias in meta-analysis detected by a simple, graphical test. BMJ 1997;315:629-34.

100. Begg CB, Mazumdar M. Operating characteristics of a rank correlation test for publication bias. Biometrics 1994;50:1088-101.

101. Duval S, Tweedie R. Trim and fill: a simple funnel-plot-based method of testing and adjusting for publication bias in meta-analysis. Biometrics 2000;56:455-63.

102. Bystritsky A, Kaplan JT, Feusner JD, et al. A preliminary study of fMRI-guided rTMS in the treatment of generalized anxiety disorder. J Clin Psychiatry 2008;69:1092-8.

103. Clarke E, Clarke P, Gill S, et al. Efficacy of repetitive transcranial magnetic stimulation in the treatment of depression with comorbid anxiety disorders. J Affect Disord 2019;252:435-9.

104. Kumar S, Singh S, Parmar A, et al. Effect of high-frequency repetitive transcranial magnetic stimulation (rTMS) in patients with comorbid panic disorder and major depression. Australas Psychiatry 2018;26: 398-400.

105. Lu R, Zhang C, Liu Y, et al. The effect of bilateral low-frequency rTMS over dorsolateral prefrontal cortex on serum brain-derived neurotropic factor and serotonin in patients with generalized anxiety disorder. Neurosci Lett 2018;684:67-71.

106. Mantovani A, Lisanby SH, Pieraccini F, et al. Repetitive transcranial magnetic stimulation (rTMS) in the treatment of panic disorder (PD) with comorbid major depression. J Affect Disord 2007;102: 277-80.

107. White D, Tavakoli S. Repetitive transcranial magnetic stimulation for treatment of major depressive disorder with comorbid generalized anxiety disorder. Ann Clin Psychiatry 2015;27:192-6.

108. Assaf M, Rabany L, Zertuche L, et al. Neural functional architecture and modulation during decision making under uncertainty in individuals with generalized anxiety disorder. Brain Behav 2018; 8:e01015.

109. Deppermann S, Notzon S, Kroczek Aet al. Functional co-activation within the prefrontal cortex supports the maintenance of behavioural performance in fear-relevant situations before an iTBS modulated virtual reality challenge in participants with spider phobia. Behav Brain Res 2016;307:208-17.

110. Deppermann S, Vennewald N, Diemer J, et al. Neurobiological and clinical effects of fNIRS-controlled rTMS in patients with panic disorder/agoraphobia during cognitive-behavioural therapy. Neuroimage Clin 2017;16:668-77.

111. Diefenbach GJ, Assaf M, Goethe JW, et al. Improvements in emotion regulation following repetitive transcranial magnetic stimulation for generalized anxiety disorder. J Anxiety Disord 2016;43:1-7.

112. Diefenbach GJ, Rabany L, Hallion LS, et al. Sleep improvements and associations with default mode network functional connectivity following rTMS for generalized anxiety disorder. Brain Stimul 2019;12:184-6.

113. Heeren A, Billieux J, Philippot $P$, et al. Impact of transcranial direct current stimulation on attentional bias for threat: a proof-ofconcept study among individuals with social anxiety disorder. Soc Cogn Affect Neurosci 2017;12:251-60. 
114. $\mathrm{Wu} \mathrm{H}, \mathrm{Hu} \mathrm{M}, \mathrm{Yu}$ B, et al. Repetitive transcranial magnetic stimulation combined with venlafaxine and lorazepam for treatment of generalized anxiety. Shanghai Jingshen Yixue 2016;28:212-8.

115. Deppermann S, Vennewald N, Diemer J et al. Does rTMS alter neurocognitive functioning in patients with panic disorder/agoraphobia? An fNIRS-based investigation of prefrontal activation during a cognitive task and its modulation via sham-controlled rTMS. BioMed Res Int 2014;2014:542526.

116. Diefenbach GJ, Bragdon LB, Zertuche L, et al. Repetitive transcranial magnetic stimulation for generalised anxiety disorder: a pilot randomised, double-blind, sham-controlled trial. Br J Psychiatry 2016209:222-8

117. Herrmann MJ, Katzorke A, Busch Y, et al. Medial prefrontal cortex stimulation accelerates therapy response of exposure therapy in acrophobia. Brain Stimul 2017;10:291-7.

118. Huang $Z$, Li $Y$, Bianchi MT, et al. Repetitive transcranial magnetic stimulation of the right parietal cortex for comorbid generalized anxiety disorder and insomnia: a randomized, double-blind, sham-controlled pilot study. Brain Stimul 2018;11:1103-9.

119. Mantovani A, Aly M, Dagan Y, et al. Randomized sham controlled trial of repetitive transcranial magnetic stimulation to the dorsolateral prefrontal cortex for the treatment of panic disorder with comorbid major depression. J Affect Disord 2013;144:153-9.

120. Movahed FS, Goradel JA, Pouresmali A, et al. Effectiveness of transcranial direct current stimulation on worry, anxiety, and depression in generalized anxiety disorder: a randomized, singleblind pharmacotherapy and sham-controlled clinical trial. Iran J Psychiatry Behav Sci 2018;12:e11071.

121. Nasiri F, Mashhadi A, Bigdeli I, et al. Augmenting the unified protocol for transdiagnostic treatment of emotional disorders with transcranial direct current stimulation in individuals with generalized anxiety disorder and comorbid depression: a randomized controlled trial. J Affect Disord 2020;262:405-13.

122. Prasko J, Záleský R, Bares M, et al. The effect of repetitive transcranial magnetic stimulation (rTMS) add on serotonin reuptake inhibitors in patients with panic disorder: a randomized, double blind sham controlled study. Neuroendocrinol Lett 2007;28:33-8.

123. Ellard KK, Fairholme CP, Boisseau CL, et al. Unified protocol for the transdiagnostic treatment of emotional disorders: protocol development and initial outcome data. Cogn Behav Pract 2010;17:88-101.

124. D'Urso G, Mantovani A, Micillo M, et al. Transcranial direct current stimulation and cognitive-behavioral therapy: evidence of a synergistic effect in treatment-resistant depression. Clin Res Neuromodulation 2013;6:465-7.

125. Gandiga PC, Hummel FC, Cohen LG. Transcranial DC stimulation (tDCS): a tool for double-blind sham-controlled clinical studies in brain stimulation. Clin Neurophysiol 2006;117:845-50.

126. Shear MK, Brown TA, Barlow DH, et al. Multicenter collaborative panic disorder severity scale. Am J Psychiatry 1997;154:1571-5.

127. Bandelow, B. Assessing the efficacy of treatments for panic disorder and agoraphobia: II. The panic and agoraphobia scale. Int Clin Psychopharmacol 1995;10:73-81.

128. Klorman R, Weerts TC, Hastings JE, et al. Psychometric description of some specific-fear questionnaires. Behav Ther 1974;5:401-9.

129. Watts FN, Sharrock R. Questionnaire dimensions of spider phobia. Behav Res Ther 1984;22:575-80.

130. Cohen DC. Comparison of self-report and overt-behavioral procedures for assessing acrophobia. Behav Ther 1977;8:17-23.

131. Meyer TJ, Miller ML, Metzger RL, et al. Development and validation of the Penn State worry questionnaire. Behav Res Ther 1990;28: 487-95.

132. Newman MG, Zuellig AR, Kachin KE, et al. Preliminary reliability and validity of the Generalized Anxiety Disorder QuestionnaireIV: a revised self-report diagnostic measure of generalized anxiety disorder. Behav Ther 2002;33:215-33.

133. Lipp MEN. Manual do inventário de sintomas de stress para adultos de Lipp (ISSL). São Paulo, Brazil: São Paulo Casa do Psicólogo; 2000.

134. Buysse DJ, Reynolds CF, Monk TH, et al. The Pittsburgh Sleep Quality Index: a new instrument for psychiatric practice and research. Psychiatry Res 1989;28:193-213.
135. Hamilton $\mathrm{M}$. The assessment of anxiety states by rating. $\mathrm{Br} J \mathrm{Med}$ Psychol 1959;32:50-5.

136. Beck AT, Epstein N, Brown G, et al. An inventory for measuring clinical anxiety: psychometric properties. J Consult Clin Psychol 1988;56:893.

137. Hamilton M. A rating scale for depression. J Neurol Neurosurg Psychiatry 1960;23:56-62.

138. Beck AT, Ward CH, Mendelson M, et al. An inventory for measuring depression. Arch Gen Psychiatry 1961;4:561-71.

139. Brown C, Schulberg HC, Madonia MJ. Assessment depression in primary care practice with the Beck Depression Inventory and the Hamilton Rating Scale for Depression. Psychol Assess 1995;7:59.

140. Baujat B, Mahé C, Pignon J, et al. A graphical method for exploring heterogeneity in meta-analyses: application to a meta-analysis of 65 trials. Stat Med 2002;21:2641-52.

141. Milev RV, Giacobbe P, Kennedy SH, et al. Canadian Network for Mood and Anxiety Treatments (CANMAT) 2016 clinical guidelines for the management of adults with major depressive disorder: section 4. Neurostimulation treatments. Can J Psychiatry 2016;61: 561-75.

142. Moffa AH, Martin D, Alonzo A, et al. Efficacy and acceptability of transcranial direct current stimulation (tDCS) for major depressive disorder: sn individual patient data meta-analysis. Prog Neuropsychopharmacol Biol Psychiatry 2020;99:109836.

143. Brunoni AR, Chaimani A, Moffa A, et al. Repetitive transcranial magnetic stimulation for the acute treatment of major depressive episodes: a systematic review with network meta-analysis. JAMA Psychiatry 2017;74:143-52.

144. Razza LB, Palumbo P, Moffa AH, et al. A systematic review and meta-analysis on the effects of transcranial direct current stimulation in depressive episodes. Depress Anxiety 2020;37:594-608.

145. McClintock SM, Reti IM, Carpenter LL, et al. Consensus recommendations for the clinical application of repetitive transcranial magnetic stimulation (rTMS) in the treatment of depression. J Clin Psychiatry 2018;79:16cs10905.

146. Yip AG, George MS, Tendler A, et al. $61 \%$ of unmedicated treatment resistant depression patients who did not respond to acute TMS treatment responded after four weeks of twice weekly deep TMS in the Brainsway pivotal trial. Brain Stimul 2017;10:847-9.

147. Bortoletto M, Pellicciari MC, Rodella C, et al. The interaction with task-induced activity is more important than polarization: a tDCS study. Brain Stimul 2015;8:269-76.

148. Romero Lauro, LJ, Pisoni A, Rosanova M, et al. Localizing the effects of anodal tDCS at the level of cortical sources: a reply to Bailey et al., 2015. Cortex 2016;74:323-8.

149. Lauro LJR, Rosanova M, Mattavelli G, et al. TDCS increases cortical excitability: direct evidence from TMS-EEG. Cortex 2014;58: 99-111.

150. Luber B, Lisanby SH. Enhancement of human cognitive performance using transcranial magnetic stimulation (TMS). Neuroimage 2014;85:961-70.

151. Romei V, Thut G, Silvanto J. Information-based approaches of noninvasive transcranial brain stimulation. Trends Neurosci 2016;39: 782-95.

152. Silvanto J, Bona S, Marelli M, et al. On the mechanisms of transcranial magnetic stimulation (TMS): how brain state and baseline performance level determine behavioral effects of TMS. Front Psychol 2018;9:741.

153. Moody TD, Morfini F, Cheng G, et al. Mechanisms of cognitivebehavioral therapy for obsessive-compulsive disorder involve robust and extensive increases in brain network connectivity. Transl Psychiatry 2017;7:e1230.

154. Yoshimura S, Okamoto Y, Matsunaga M, et al. Cognitive behavioral therapy changes functional connectivity between medial prefrontal and anterior cingulate cortices. J Affect Disord 2017;208:610-4.

155. Breining BL, Sebastian R. Neuromodulation in post-stroke aphasia treatment. Curr Phys Med Rehabil Rep 2020;8:44-56.

156. Pruski A, Cantarero G. Transcranial direct current stimulation for motor recovery following brain injury. Curr Phys Med Rehabil Rep 2020;8:268-79. 
157. Tsagaris KZ, Labar DR, Edwards DJ. A framework for combining rTMS with behavioral therapy. Front Syst Neurosci 2016;10:82.

158. Segrave RA, Arnold S, Hoy K, et al. Concurrent cognitive control training augments the antidepressant efficacy of tDCS: a pilot study. Brain Stimul 2014;7:325-31.

159. Jahshan C, Rassovsky Y, Green MF. Enhancing neuroplasticity to augment cognitive remediation in schizophrenia. Front Psychiatry 2017;8:191

160. Polania R, Nitsche MA, Ruff CC. Studying and modifying brain function with non-invasive brain stimulation. Nat Neurosci 2018;21:174-87.

161. Carmi L, Alyagon U, Barnea-Ygael N, et al. Clinical and electrophysiological outcomes of deep TMS over the medial prefrontal and anterior cingulate cortices in OCD patients. Brain Stimul 2018;11:158-65.

162. Carmi L, Tendler A, Bystritsky A, et al. Efficacy and safety of deep transcranial magnetic stimulation for obsessive-compulsive disorder: a prospective multicenter randomized double-blind placebocontrolled trial. Am J Psychiatry 2019;176:931-8.

163. Gellersen HM, Kedzior KK. Antidepressant outcomes of highfrequency repetitive transcranial magnetic stimulation (rTMS) with F8-coil and deep transcranial magnetic stimulation (DTMS) with H1-coil in major depression: a systematic review and metaanalysis. BMC Psychiatry 2019;19:139.

164. Roth Y, Amir A, Levkovitz Y, et al. Three-dimensional distribution of the electric field induced in the brain by transcranial magnetic stimulation using figure-8 and deep H-coils. J Clin Neurophysiol 2007;24:31-8

165. Rakofsky JJ, Holtzheimer PE, Nemeroff CB. Emerging targets for antidepressant therapies. Curr Opin Chem Biol 2009;13:291-302.

166. Stehberg J, Levy D, Zangen A. Impairment of aversive memory reconsolidation by localized intracranial electrical stimulation. Eur J Neurosci 2009;29:964-9.

167. Berlim MT, et al. Augmenting antidepressants with deep transcranial magnetic stimulation (DTMS) in treatment-resistant major depression. World J Biol Psychiatry 2014;15:570-8

168. Chen L, Hudaib A, Hoy KE, et al. Is rTMS effective for anxiety symptoms in major depressive disorder? An efficacy analysis comparing left-sided high-frequency, right-sided low-frequency, and sequential bilateral rTMS protocols. Depress Anxiety 2019;36: 723-31.

169. Uchida H, Hirao K. Prefrontal cortex hypoactivity distinguishes severe from mild-to-moderate social anxiety as revealed by a palmsized near-infrared spectroscopy system. J Neural Transm (Vienna) 2020;127:1305-13

170. Gilio F, Rizzo V, Siebner HR, et al. Effects on the right motor hand-area excitability produced by low-frequency rTMS over human contralateral homologous cortex. J Physiol 2003;551:563-73.
171. Smirni D, Turriziani P, Mangano GR, et al. Modulating memory performance in healthy subjects with transcranial direct current stimulation over the right dorsolateral prefrontal cortex. PLoS One 2015;10:e0144838.

172. Vergallito A, Lauro R, Bonandrini R, et al. What is difficult for you can be easy for me. Effects of increasing individual task demand on prefrontal lateralization: a tDCS study. Neuropsychologia 2018;109:283-94

173. Otal B, Olma MC, Flöel A, et al. Inhibitory non-invasive brain stimulation to homologous language regions as an adjunct to speech and language therapy in post-stroke aphasia: a metaanalysis. Front Hum Neurosci 2015;9:236.

174. Bertolucci F, Chisari C, Fregni F. The potential dual role of transcallosal inhibition in post-stroke motor recovery. Restor Neurol Neurosci 2018;36:83-97.

175. Maggioni E, Delvecchio G, Grottaroli M, et al. Common and different neural markers in major depression and anxiety disorders: apilot structural magnetic resonance imaging study. Psychiatry Res Neuroimaging 2019;290:42-50.

176. Morawetz C, Bode S, Derntl B, et al. The effect of strategies, goals and stimulus material on the neural mechanisms of emotion regulation: a meta-analysis of fMRI studies. Neurosci Biobehav Rev 2017;72:111-28.

177. Phillips ML, Ladouceur CD, Drevets WC. A neural model of voluntary and automatic emotion regulation: implications for understanding the pathophysiology and neurodevelopment of bipolar disorder. Mol Psychiatry 2008;13:833-57.

178. Vergallito A, Riva P, Pisoni A, et al. Modulation of negative emotions through anodal tDCS over the right ventrolateral prefrontal cortex. Neuropsychologia 2018;119:128-135.

179. Baumert A, Buchholz N, Zinkernagel A, et al. Causal underpinnings of working memory and Stroop interference control: testing the effects of anodal and cathodal tDCS over the left DLPFC. Cogn Affect Behav Neurosci 2020;20:34-48.

180. Ironside M, O'Shea J, Cowen PJ, et al. Frontal cortex stimulation reduces vigilance to threat: implications for the treatment of depression and anxiety. Biol Psychiatry 2016;79:823-30.

181. Stein DJ, Medeiros LF, Caumo W, et al. Transcranial direct current stimulation in patients with anxiety: current perspectives. Neuropsychiatr Dis Treat 2020;16:161.

182. Norrholm SD, Jovanovic T, Gerardi M, et al. Baseline psychophysiological and cortisol reactivity as a predictor of PTSD treatment outcome in virtual reality exposure therapy. Behav Res Ther 2016;82:28-37.

183. Dawood AB, Dickinson A, Aytemur A, et al. Investigating the effects of tDCS on visual orientation discrimination task performance: the possible influence of placebo. J Cogn Enhanc 2020;4:235-49.

184. Turi Z, Bjørkedal E, Gunkel L, et al. Evidence for cognitive placebo and nocebo effects in healthy individuals. Sci Rep 2018;8:17443. 